Cahiers du MONDE RUSSE

\section{Cahiers du monde russe}

Russie - Empire russe - Union soviétique et États indépendants

$55 / 1-2 \mid 2014$

Fiscalité, justice et conflit politique en Russie, premier tiers du XVIIle siècle

\title{
КАНЦЕЛЯРИИ СБОРА ПОДУШНЫХ ДЕНЕГ
}

СТАТУС И МЕСТО В АДМИНИСТРАТИВНОЙ СИСТЕМЕ РОССИИ (ПЕРВАЯ TPETЬ XVIII В.)

Statut et rôle des bureaux de collecte de l'impôt de capitation dans le système administratif de la Russie durant le premier tiers du XVIII siècle

Status and role of poll tax collection bureaus in the Russian administrative system in the first third of the eighteenth century

Dmitrij A. Redin

\section{Q OpenEdition}

1 Journals

\section{Édition électronique}

URL : http://journals.openedition.org/monderusse/7984

DOI : $10.4000 /$ monderusse.7984

ISSN : $1777-5388$

Éditeur

Éditions de l'EHESS

\section{Édition imprimée}

Date de publication : 1 janvier 2014

Pagination : 51-70

ISBN : 9782713224409

ISSN : 1252-6576

Référence électronique

Dmitrij A. Redin, «Канцелярии сбора подушных денег », Cahiers du monde russe [Онлайн],

55/1-2 | 2014, Выложить онлайн 01 janvier 2017, Наводить справки в 02 mai 2019. URL' : http:// journals.openedition.org/monderusse/7984; DOI : 10.4000/monderusse.7984

Ce document a été généré automatiquement le 2 mai 2019. 


\title{
КАНЦЕЛЯРИИ СБОРА ПОДУШНЫХ
}

\section{ДЕНЕГ}

\author{
СТАТУС И МЕСТО В АДМИНИСТРАТИВНОЙ СИСТЕМЕ РОССИИ (ПЕРВАЯ \\ ТРЕТЬ XVIII В.)
}

Statut et rôle des bureaux de collecte de l'impôt de capitation dans le système administratif de la Russie durant le premier tiers du XVIII siècle

Status and role of poll tax collection bureaus in the Russian administrative system in the first third of the eighteenth century

\section{Dmitrij A. Redin}

1 СРЕДИ ПРЕОБРАЗОВАНИЙ, ПРЕДПРИНЯТЫХ ПЕТРОМ ВЕЛИКИМ, ОДНО ИЗ КЛЮЧЕВЫХ МЕСТ, БЕССПОРНО, ЗАНИМАЛА РЕФОРМА НАЛОГООБЛОЖЕНИЯ. КАК ИЗВЕСТНО, ЕЕ ГЛАВНОЙ ЦЕЛЬЮ БЫЛО ВВЕДЕНИЕ НОВОГО ПРЯМОГО НАЛОГА - ПОДУШНОЙ ПОДАТИ, КОТОРУЮ ПРЕДПОЛАГАЛОСЬ СОБИРАТЬ ПО ОПРЕДЕЛЕННОЙ СТАВКЕ С КАЖДОЙ ДУШИ МУЖСКОГО ПОЛА» НА СОДЕРЖАНИЕ РЕГУЛЯРНОЙ АРМИИ. СБОР НОВОГО НАЛОГА ВЛАСТИ НАМЕРЕВАЛИСЬ ВОЗЛОЖИТЬ НА САМУ АРМИЮ, РАСКВАРТИРОВАННУЮ В МИРНОЕ ВРЕМЯ ПО ГУБЕРНИЯМ, НА ТЕРРИТОРИИ КОТОРЫХ СЛЕДОВАЛО ПОСТРОИТЬ ЦЕЛУЮ СЕТЬ СПЕЦИАЛЬНЫХ ПОСЕЛЕНИЙ-СЛОБОД - « ЗИМНИХ», ИЛИ « ВЕЧНЫХ» КВАРТИР.

ПРОБЛЕМА КВАРТИРОВАНИЯ РЕГУЛЯРНОЙ АРМИИ, КОТОРУЮ, В ОТЛИЧИЕ ОТ ОПОЛЧЕНИЯ, НЕ ПРЕДПОЛАГАЛОСЬ РАСПУСКАТЬ ПО ДОМАМ В МИРНОЕ ВРЕМЯ, СТАЛА ЗАБОТИТЬ РОССИЙСКОГО МОНАРХА ВСКОРЕ ПОСЛЕ ПОЛТАВСКОЙ ПОБЕДЫ, КОГДА ПОЯВИЛАСЬ НАДЕЖДА НА ОКОНЧАНИЕ ВОЙНЫ. ИЗВЕСТНО, ЧТО ИДЕЯ ТАКОГО РАЗМЕЩЕНИЯ И СОДЕРЖАНИЯ ВОЙСК ДЛЯ СОХРАНЕНИЯ ИХ БОЕГОТОВНОСТИ В МЕЖВОЕННЫЕ ПЕРИОДЫ БЫЛА ВОСПРИНЯТА ЦАРЕМ ИЗ ШВЕДСКОГО ОПЫТА ${ }^{1}$, ХОТЯ ЕЩЕ В 1705 Г. О ЧЕМ-ТО ПОХОЖЕМ НА ШВЕДСКУЮ СИСТЕМУ СООБЩАЛ ГОСУДАРЮ ПУТЕШЕСТВОВАВШИЙ ПО ЕВРОПЕ А.А. МАТВЕЕВ, ССЫЛАЯСЬ НА ПРУССКУЮ ПРАКТИКУ². ВПРОЧЕМ, КАК СПРАВЕДЛИВО ПИСАЛИ ЕЩЕ ДОРЕВОЛЮЦИОННЫЕ ИСТОРИКИ, ПЕТР МОГ ПОЧЕРПНУТЬ ИЗ ШВЕДСКОГО ОПЫТА ИМЕННО И ТОЛЬКО ЛИШЬ САМУ ИДЕЮ ТЕРРИТОРИАЛЬНОГО РАСПОЛОЖЕНИЯ И ОБЕСПЕЧЕНИЯ АРМИИ, НО НИКАК НЕ ГОТОВЫЕ РЕЦЕПТЫ ЕЕ РЕАЛИЗАЦИИ. 
ПО ОБЩЕМУ МНЕНИЮ ИСТОРИКОВ, ВВЕДЕНИЕ ПОДУШНОЙ ПОДАТИ ОКАЗАЛО ВЛИЯНИЕ НЕ ТОЛЬКО НА ФИНАНСОВУЮ СИСТЕМУ И ЭКОНОМИКУ РОССИИ. РЕФОРМА ИМЕЛА ШИРОКИЕ СОЦИАЛЬНЫЕ ПОСЛЕДСТВИЯ, ПОСКОЛЬКУ В ХОДЕ ЕЕ БЫЛА ЗАЛОЖЕНА ОСНОВА НОВОЙ МОДЕЛИ УЧЕТА НАСЕЛЕНИЯ, УСИЛЕНЫ ПРОЦЕССЫ СОСЛОВНОЙ КОНСОЛИДАЦИИ, УКРЕПЛЕН АДМИНИСТРАТИВНЫЙ КОНТРОЛЬ НАД ОБЩЕСТВОМ, СТИМУЛИРОВАНО РАЗВИТИЕ КРЕПОСТНИЧЕСТВА И Т.П. КРОМЕ ТОГО, ПОДАТНАЯ РЕФОРМА СЕРЬЕЗНО ПОВЛИЯЛА НА СИСТЕМУ ГОСУДАРСТВЕННОГО УПРАВЛЕНИЯ РОССИЙСКИЙ ИМПЕРИИ.

РАДИЦИОННО ВОПРОСЫ ФИСКА НАХОДИЛИСЬ В СФЕРЕ КОМПЕТЕНЦИИ ГРАЖДАНСКИХ АДМИНИСТРАЦИЙ ОБЩЕГО УПРАВЛЕНИЯ. ПРАКТИЧЕСКИ ОДНОВРЕМЕННО С РАЗРАБОТКОЙ И ПРОВЕДЕНИЕМ НАЧАЛЬНОГО ЭТАПА ПОДАТНОЙ РЕФОРМЫ (ПЕРЕПИСЬ МУЖСКОГО НАСЕЛЕНИЯ 1719 Г.), В СТРАНЕ РАЗВЕРНУЛИСЬ Т.Н. КОЛЛЕЖСКАЯ И ВТОРАЯ ОБЛАСТНАЯ РЕФОРМЫ, В ХОДЕ КОТОРЫХ НАЛОГОВЫЕ СБОРЫ ПЕРЕДАВАЛИСЬ В ВЕДЕНИЕ СПЕЦИАЛИЗИРОВАННЫХ ОРГАНОВ, ПОДЧИНЕННЫХ КАМЕР-КОЛЛЕГИИ: КАНЦЕЛЯРИЙ ЗЕМСКИХ КОМИССАРОВ-РУКОВОДИТЕЛЕЙ «КАМЕР-КОЛЛЕЖСКИХ» ДИСТРИКТОВ (УЕЗДНЫЙ УРОВЕНЬ) И КАМЕРИРСКИХ КОНТОР ВО ГЛАВЕ С ЗЕМСКИМИ КАМЕРИРАМИ (ПРОВИНЦИАЛЬНЫЙ/ГУБЕРНСКИЙ УРОВЕНЬ). Т.О., ВВОДЯ НОВЫЙ ПРЯМОЙ НАЛОГ И ДОВЕРЯЯ ЕГО СБОР ВОЕННЫМ, ВЛАСТИ, КАК МИНИМУМ, ДОЛЖНЫ БЫЛИ РЕШИТЬ, КАКОВ БУДЕТ АДМИНИСТРАТИВНЫЙ МЕХАНИЗМ ВЗИМАНИЯ ЭТОГО НАЛОГА И КАКИМ ОБРАЗОМ ОН БУДЕТ СООТНОСИТЬСЯ С ПАРАЛЛЕЛЬНО РЕОРГАНИЗУЕМОЙ ОБЩЕАДМИНИСТРАТИВНОЙ СИСТЕМОЙ УПРАВЛЕНИЯ. В СИЛУ СКАЗАННОГО, ПРОБЛЕМА КВАРТИРОВАНИЯ И СОДЕРЖАНИЯ ВОЙСК ЗА СЧЕТ ПОДУШНОЙ ПОДАТИ СТАНОВИЛАСЬ, ПОМИМО ПРОЧЕГО, УПРАВЛЕНЧЕСКОЙ ЗАДАЧЕЙ.

КАК РЕШАЛАСЬ ЭТА ЗАДАЧА? КАКИЕ ИМЕННО ВОЕННЫЕ СТРУКТУРЫ БЫЛИ ОТВЕТСТВЕННЫ ЗА СБОР ПОДУШНОЙ ПОДАТИ ? В КАКИХ ОТНОШЕНИЯХ ОНИ НАХОДИЛИСЬ С ОРГАНАМИ ГРАЖДАНСКОГО ОБЩЕГО И СПЕЦИАЛЬНОГО УПРАВЛЕНИЯ ? КАК НИ ПАРАДОКСАЛЬНО, ЛИТЕРАТУРА, ПОСВЯЩЕННАЯ ЭТОЙ ТЕМАТИКЕ, КРАЙНЕ СКУДНАЗ ЕДИНСТВЕННЫМ СИСТЕМАТИЧЕСКИМ ИЗЛОЖЕНИЕМ ИСТОРИИ ВОЕННО-ПОДАТНЫХ УЧРЕЖДЕНИЙ КАК ЭЛЕМЕНТА ГОСУДАРСТВЕННОГО АППАРАТА ДО СИХ ПОР ОСТАЕТСЯ ПЯТАЯ ГЛАВА ФУНДАМЕНТАЛЬНОЙ МОНОГРАФИИ МИХАИЛА МИХАЙЛОВИЧА БОГОСЛОВСКОГО ОБЛАСТНАЯ РЕФОРМА ПЕТРА ВЕЛИКОГО: ПРОВИНЦИЯ 1719-1727 ГГ, УВИДЕВШАЯ СВЕТ В $1902 Г{ }^{4}$ НАДО ВОЗДАТЬ ДОЛЖНОЕ АВТОРУ: ТЩАТЕЛЬНОСТЬ ИСТОЧНИКОВЕДЧЕСКИХ РЕКОНСТРУКЦИЙ И НАУЧНАЯ ДОБРОСОВЕСТНОСТЬ, ПРИСУЩИЕ ВСЕМУ ТВОРЧЕСТВУ БОГОСЛОВСКОГО, СДЕЛАЛИ ЭТОТ ОЧЕРК ИСТОРИИ ВОЕННО-ФИСКАЛЬНЫХ ОРГАНОВ НАДЕЖНЫМ ФУНДАМЕНТОМ ДЛЯ БУДУЩИХ ПОСТРОЕНИЙ. ЕГО ЗНАЧЕНИЕ ВЕЛИКО ПО СЕЙ ДЕНЬ, А СОБРАННЫЙ И СКОМПОНОВАННЫЙ УЧЕНЫМ МАТЕРИАЛ МОЖЕТ БЫТЬ ПОПОЛНЕН ТОЛЬКО В РЕЗУЛЬТАТЕ ОТДЕЛЬНОГО МОНОГРАФИЧЕСКОГО ИССЛЕДОВАНИЯ ДАННОЙ ТЕМЫ. ОДНАКО ДАЖЕ ИМЕЮЩИЕСЯ В НАШЕМ РАСПОРЯЖЕНИИ МАТЕРИАЛЫ, ПО-НОВОМУ ПРОИНТЕРПРЕТИРОВАННЫЕ, ПОЗВОЛЯЮТ СУЩЕСТВЕННО СКОРРЕКТИРОВАТЬ ПРЕДСТАВЛЕНИЯ О ВЛИЯНИИ ПОДАТНОЙ РЕФОРМЫ НА АДМИНИСТРАТИВНЫЙ ЛАНДШАФТ ПОСЛЕДНИХ ЛЕТ ЦАРСТВОВАНИЯ ПЕТРА І. ПЫТАЯСЬ ОСМЫСЛИТЬ В ЕДИНОМ КОНТЕКСТЕ РЕАЛИЗАЦИЮ ВТОРОЙ ОБЛАСТНОЙ И ПОДАТНОЙ РЕФОРМ, БОГОСЛОВСКИЙ, В ЧАСТНОСТИ, ПРИШЕЛ К ВЫВОДУ, ЧТО ОНИ « ОБСУЖДАЛИСЬ И ОСУЩЕСТВЛЯЛИСЬ ОДНОВРЕМЕННО И ПАРАЛЛЕЛЬНО, НО ПОРОЗНЬ И БЕЗ МАЛЕЙШЕЙ ПОПЫТКИ СОГЛАСОВАТЬ ОДНУ С ДРУГОЙ» ОЦЕНКА НЕ ВПОЛНЕ ОТРАЖАЕТ РЕАЛЬНОСТЬ. НЕ ОСТАВЛЯЯ НАДЕЖДЫ ГАРМОНИЧНО 
ОБЪЕДИНИТЬ ГОСУДАРСТВЕННОЕ УПРАВЛЕНИЕ, ФИСК И АРМИЮ, ЦАРЬ, СУДЯ ПО ВСЕМУ, НАПРЯЖЕННО РАБОТАЛ НАД НЕКИМ ВАРИАНТОМ ТАКОГО СИНТЕЗА. ПЕРВЫЕ РЕЗУЛЬТАТЫ ЕГО ТРУДОВ МОЖНО УСМОТРЕТЬ В ДВУХ ИМЕННЫХ УКАЗАХ, ОПУБЛИКОВАННЫХ ОДНОВРЕМЕННО, 26 НОЯБРЯ 1718 Г.

7 ОДИН ИЗ ЭТИХ УКАЗОВ ПРЕДПОЛАГАЛ ВВЕДЕНИЕ В ГУБЕРНИЯХ НОВЫХ АДМИНИСТРАТИВНЫХ ДОЛЖНОСТЕЙ : В КАЧЕСТВЕ ЭКСПЕРИМЕНТА - В САНКТ-ПЕТЕРБУРГСКОЙ ГУБЕРНИИ - С 1719 Г., А ПОВСЕМЕСТНО - С 1720 Г. $^{6}$ ВНОВЬ ОПРЕДЕЛЕННЫЕ ОБЛАСТНЫЕ ЧИНОВНИКИ ДОЛЖНЫ БЫЛИ ПОЛУЧИТЬ СЛУЖЕБНЫЕ « ИНСТРУКЦИИ И ПРОТЧИЕ ПОРЯДКИ ВСЕ ПРОТИВ ШВЕДСКОГО (ИЛИ ЧТО ПЕРЕПРАВЯ)» ДО КОНЦА 1718 Г. ПРИВЕДЕННАЯ В УКАЗЕ «РОСПИСЬ ЧИНАМ» РАСКРЫВАЛА СТРУКТУРУ РУКОВОДЯЩЕГО И СТАРШЕГО КАНЦЕЛЯРСКОГО СОСТАВА МЕСТНЫХ УЧРЕЖДЕНИЙ. ШВЕДСКИЕ НАЗВАНИЯ ДОЛЖНОСТЕЙ СОПРОВОЖДАЛИСЬ РУССКИМИ ТОЛКОВАНИЯМИ. ИЗ ДВЕНАДЦАТИ ЧИНОВ РОСПИСИ, ВОСЕМЬ, КАК МОЖНО ПОНЯТЬ, ПРЕДСТАВЛЯЛИ ГУБЕРНСКИЙ ШТАТ. МЕСТНУЮ ИЕРАРХИЮ ДОЛЖЕН БЫЛ ВОЗГЛАВИТЬ ЛАНДСГЕВДИНГ, «ГОЛОВА ЗЕМСКОЙ», КАК ОБЪЯСНЯЛСЯ ЭТОТ РАНГ В ТЕКСТЕ. ЛАНДСГЕВДИНГОМ НАЗЫВАЛСЯ ГЛАВА ШВЕДСКОЙ ГУБЕРНИИ-ЛЕНА (« ЛАНДСГЕВДИНГСТВА») СООТВЕТСТВЕННО, ТАК ДОЛЖНЫ БЫЛИ ВПРЕДЬ ИМЕНОВАТЬСЯ РАНЕЕ СУЩЕСТВОВАВШИЕ В РОССИИ ГУБЕРНАТОРЫ. СЛЕДУЮЩИЙ ЧИН РОСПИСИ - ОБЕР-ЛАНДРИХТЕР, ИЛИ « ВЫШНИЙ ЗЕМСКИЙ СУДЬЯ» - БЫЛ УЖЕ ПРИВЫЧЕН ОТЕЧЕСТВЕННОЙ АДМИНИСТРАТИВНОЙ ПРАКТИКЕ, ПОСКОЛЬКУ, КАК ИЗВЕСТНО, ЛАНДРИХТЕРЫ СУЩЕСТВОВАЛИ В МЕСТНОМ АППАРАТЕ УПРАВЛЕНИЯ В 1710-Х ГГ. ЦИТИРУЕМЫЙ УКАЗ ЛИШЬ ПОДЧЕРКИВАЛ ОСОБЫЕ СУДЕБНЫЕ ПОЛНОМОЧИЯ ОБЕР-ЛАНДРИХТЕРА НОВОГО ОБРАЗЦА. ЗАМЫКАЛ ПЕРВУЮ ТРОЙКУ ГУБЕРНСКИХ ЧИНОВ ЛАНД-СЕКРЕТАРЬ (« ЗЕМСКОЙ ДЬЯК») - ГЛАВА ГУБЕРНСКОЙ КАНЦЕЛЯРИИ. ЕЩЕ ПЯТЬ ЧИНОВНИКОВ ДОЛЖНЫ БЫЛИ ОТВЕЧАТЬ ЗА РАЗЛИЧНЫЕ СФЕРЫ ГУБЕРНСКОГО УПРАВЛЕНИЯ : БУХГАЛТЕР («ЗЕМСКОЙ НАДЗИРАТЕЛЬ СБОРОВ»), ЛАНД-РЕНТМЕЙСТЕР (« ЗЕМСКОЙ КАЗНАЧЕЙ»), ЛАНД-ФИСКАЛ («ЗЕМСКОЙ ФИСКАЛ»), ЛАНД-МЕССЕР (« МЕЖЕ-В-ЩИК») И ПРОФОС (« СТАРОСТА ТЮРЕМНЫЙ»). СЛЕДУЮЩИЕ ТРИ ДОЛЖНОСТИ ПРЕДСТАВЛЯЛИ СРЕДНИЙ УРОВЕНЬ МЕСТНОГО УПРАВЛЕНИЯ. ЛАНД-КОМИССАР («ЗЕМСКОЙ КОМИССАР»), ЛАНДРИХТЕР (« ЗЕМСКОЙ СУДЬЯ») И ЛАНДС-ШРАЙБЕР (« ПОДЬЯЧЕЙ ЗЕМСКОЙ») МОГЛИ БЫТЬ СООТНЕСЕНЫ С УЕЗДНЫМ ИЛИ ПРОВИНЦИАЛЬНЫМ ЗВЕНОМ. НА БОЛЕЕ НИЗКОЙ СТУПЕНИ АДМИНИСТРАЦИИ ОНИ СОСТАВЛЯЛИ АНАЛОГИЮ ЛАНДСГЕВДИНГУ, ОБЕР-ЛАНДРИХТЕРУ И ЛАНД-СЕКРЕТАРЮ. НАКОНЕЦ, НА СУЩЕСТВОВАНИЕ ТРЕТЬЕГО, НИЗШЕГО УРОВНЯ УПРАВЛЕНИЯ, УКАЗЫВАЕТ УПОМИНАНИЕ В РОСПИСИ ДОЛЖНОСТИ КИРХШПИЛЬ-ФОХТА, РУССКОГО ТОЛКОВАНИЯ КОТОРОЙ НЕ ПРИВОДИЛОСЬ. МЕЖДУ ТЕМ, ИЗВЕСТНО, ЧТО В ШВЕДСКОЙ СИСТЕМЕ МЕСТНОГО УПРАВЛЕНИЯ КИРХШПИЛЬФОХТ БЫЛ ГЛАВОЙ ПЕРВИЧНОЙ, НИЗШЕЙ АДМИНИСТРАТИВНО-ТЕРРИТОРИАЛЬНОЙ ЕДИНИЦЫ В ТРЕХСТУПЕНЧАТОЙ СИСТЕМЕ ТЕРРИТОРИАЛЬНОГО ДЕЛЕНИЯ КОРОЛЕВСТВА - КИРХШПИЛЯ (ЦЕРКОВНОГО ПРИХОДА)

ОТСЮДА МОЖНО ПРЕДПОЛОЖИТЬ, ЧТО В УПОМЯНУТОМ УКАЗЕ ОТ 26 НОЯБРЯ 1718 Г. ТОЖЕ ЗАКЛАДЫВАЛАСЬ ТРЕХСТУПЕНЧАТАЯ ОРГАНИЗАЦИЯ МЕСТНОЙ ВЛАСТИ. ИЗ СОДЕРЖАНИЯ ЭТОГО ДОКУМЕНТА СОВЕРШЕННО ОПРЕДЕЛЕННО ВИДНО СТРЕМЛЕНИЕ РЕФОРМАТОРА СПЕЦИАЛИЗИРОВАТЬ УПРАВЛЕНИЕ. СПЕЦИАЛИЗАЦИЯ НАИБОЛЕЕ ДЕТАЛЬНО ПРОРАБОТАНА ДЛЯ ВЫСШЕГО - ГУБЕРНСКОГО - УРОВНЯ. В « РОСПИСИ ЧИНАМ» МОЖНО ОТМЕТИТЬ СУДЕБНЫЕ, ФИНАНСОВЫЕ, НАДЗОРНЫЕ И ДАЖЕ ТАКИЕ « ЭКЗОТИЧЕСКИЕ», КАК МЕЖЕВУЮ И ТЮРЕМНУЮ ДОЛЖНОСТИ. В ГУБЕРНАТОРОМ-ЛАНДСГЕВДИНГОМ, ВЕРОЯТНО, ЗАКРЕПЛЯЛИСЬ 
ОБЩЕАДМИНИСТРАТИВНЫЕ И, БЫТЬ МОЖЕТ, ВОЕННЫЕ ФУНКЦИИ. СЛЕДУЮЩИЙ УРОВЕНЬ ВЛАСТИ ХАРАКТЕРИЗОВАЛСЯ МЕНЬШЕЙ СТЕПЕНЬЮ СПЕЦИАЛИЗАЦИИ. СПЕЦИАЛЬНОЕ УПРАВЛЕНИЕ ЗДЕСЬ ПРЕДСТАВЛЕНО ТОЛЬКО СУДЬЕЙ-ЛАНДРИХТЕРОМ, А ОРГАНИЗАЦИЯ ВЛАСТИ НИЗОВОГО ЗВЕНА, ПОХОЖЕ, НЕ ВПОЛНЕ БЫЛА ЯСНА И САМОМУ ЗАКОНОДАТЕЛЮ.

НЕКОТОРЫЕ ВЕСЬМА ВАЖНЫЕ ДОПОЛНЕНИЯ ПО ПОВОДУ ФУНКЦИОНАЛЬНОГО РАЗДЕЛЕНИЯ ПОЛНОМОЧИЙ НА НИЖНИХ ЭТАЖАХ ОБЛАСТНОЙ ВЛАСТИ ДАЕТ ВТОРОЙ ИМЕННОЙ УКАЗ, ПРИНЯТЫЙ 26 НОЯБРЯ, КОТОРЫЙ САНКЦИОНИРОВАЛ НАЧАЛО ПРОВЕДЕНИЯ ПОДАТНОЙ РЕФОРМЫ. НАПОМНЮ, ЧТО ЭТОТ АКТ, ИЗВЕСТНЫЙ В НАУЧНОМ ОБИХОДЕ КАК УКАЗ О ВВЕДЕНИИ ПОДУШНОЙ ПОДАТИ, УСТАНАВЛИВАЛ ПОРЯДОК ПРОВЕДЕНИЯ ПОДУШНОЙ ПЕРЕПИСИ ТЯГЛОГО НАСЕЛЕНИЯ, НОВЫЕ ПРИНЦИПЫ ОБЛОЖЕНИЯ И ПРОЦЕДУРЫ ВЗИМАНИЯ НАЛОГА. ОСОБЫЙ ИНТЕРЕС ПРЕДСТАВЛЯЕТ ДЛЯ НАС ТРЕТИЙ ПУНКТ УКАЗА, КОТОРЫЙ ПРЕДПОЛАГАЛ УЧРЕЖДЕНИЕ ОСОБОЙ ДОЛЖНОСТИ - ВЫБОРНОГО КОМИССАРА ОТ ЗЕМЛИ, ОТВЕТСТВЕННОГО СО СТОРОНЫ МЕСТНОЙ АДМИНИСТРАЦИИ ЗА СБОР УСТАНОВЛЕННОГО НАЛОГА. КОМИССАР ОТ ЗЕМЛИ ОЛИЦЕТВОРЯЛ СОБОЙ СБОРЩИКА ПОДУШНОЙ ПОДАТИ НА УРОВНЕ УЕЗДА (ИМЕННО УЕЗДА, Т.К. ОН, СОГЛАСНО УКАЗУ, ИЗБИРАЛСЯ УЕЗДНЫМ ДВОРЯНСТВОМ).

НИ ОДИН ИЗ УКАЗОВ ОТ 26 НОЯБРЯ 1718 Г. НЕ ДАВАЛ ТОЧНОГО ПРЕДСТАВЛЕНИЯ О СИСТЕМЕ СОПОДЧИНЕНИЯ ВСЕХ ПЕРЕЧИСЛЕННЫХ ДОЛЖНОСТНЫХ ЛИЦ. ТЕМ НЕ МЕНЕЕ, ЕСЛИ УЧЕСТЬ ОДНОВРЕМЕННОСТЬ ИХ ПОДПИСАНИЯ И НЕСОМНЕННУЮ ПРИЧАСТНОСТЬ К СОСТАВЛЕНИЮ ОБОИХ ДОКУМЕНТОВ САМОГО ЦАРЯ, ТО НЕ ПРИДЕТСЯ УСОМНИТЬСЯ В ТОМ, ЧТО ПЕРЕД НАМИ - РЕЗУЛЬТАТ ПОПЫТКИ СИСТЕМНОГО ВЗГЛЯДА НА ПРОБЛЕМУ ОРГАНИЗАЦИИ МЕСТНОГО УПРАВЛЕНИЯ, ФИСКА И СОДЕРЖАНИЯ ВОЙСК. ПРИ ВСЕХ НЕСТЫКОВКАХ И УМОЛЧАНИЯХ, СВОЙСТВЕННЫХ АДМИНИСТРАТИВНОМУ ЗАКОНОДАТЕЛЬСТВУ ПЕТРОВСКОГО ВРЕМЕНИ, ОБА УКАЗА ФОРМИРОВАЛИ ОБЩУЮ КОМПОЗИЦИЮ ВЛАСТИ, В КОТОРОЙ КОМИССАР ОТ ЗЕМЛИ СТАНОВИЛСЯ КЛЮЧЕВОЙ СВЯЗУЮЩЕЙ ФИГУРОЙ. ПО СВОЕМУ МЕСТУ В ИЕРАРХИИ УПРАВЛЕНИЯ, ОН ОКАЗЫВАЛСЯ СОПОСТАВИМ С ТРЕМЯ ЧИНАМИ СРЕДНЕЙ АДМИНИСТРАЦИИ (ЛАНД-КОМИССАРОМ, ЛАНДРИХТЕРОМ И ЛАНД-ШРАЙБЕРОМ), А ПО СФЕРЕ КОМПЕТЕНЦИИ МОГ СЧИТАТЬСЯ УЕЗДНЫМ АНАЛОГОМ ГУБЕРНСКИХ ЧИНОВ, ОТВЕТСТВЕННЫХ ЗА СБОРЫ И ФИНАНСЫ (БУХГАЛТЕРА И ЛАНД-РЕНТМЕЙСТЕРА). В СООТВЕТСТВИИ С УКАЗОМ «О ВВЕДЕНИИ ПОДУШНОЙ ПОДАТИ» УСТАНАВЛИВАЛАСЬ (ПРАВДА В САМЫХ ОБЩИХ ЧЕРТАХ) И ПОДОТЧЕТНОСТЬ КОМИССАРА ОТ ЗЕМЛИ: ЛАНДСГЕВДИНГУ, КАК ГЛАВЕ ГУБЕРНСКОЙ АДМИНИСТРАЦИИ, И ДВУМ ЦЕНТРАЛЬНЫМ ВЕДОМСТВАМ - РЕВИЗИОН- И ВОЕННОЙ КОЛЛЕГИЯМ. ЭТИМ ИНСТАНЦИЯМ КОМИССАР ОТ ЗЕМЛИ ДОЛЖЕН БЫЛ ВЕДЕНИЕ ПОДАВАТЬ» О ПЕРЕДАЧЕ СОБРАННЫХ ИМ СУММ УПОЛНОМОЧЕННОМУ КОМИССАРУ ТОГО ПОЛКА, СОДЕРЖАНИЕ КОТОРОГО ПОЛОЖЕНО НА КОНКРЕТНЫЙ УЕЗД. ТАК, НЕЗАМЕТНО, КАК БЫ МЕЖДУ ДЕЛОМ, В НЕБЕЗУПРЕЧНУЮ, НО ИНТЕНЦИОННО СИСТЕМНУЮ КОНФИГУРАЦИЮ МЕСТНОЙ ГРАЖДАНСКОЙ АДМИНИСТРАЦИИ ВОШЛИ ПОЛК И ПОЛКОВОЙ КОМИССАР - ЗАЧАТОК ВОЕННО-АДМИНИСТРАТИВНОЙ ОРГАНИЗАЦИИ В ОБЛАСТИ. ПОДОТЧЕТНОСТЬ КОМИССАРА ОТ ЗЕМЛИ ГУБЕРНАТОРУ-ЛАНДСГЕВДИНГУ ПРИВЯЗЫВАЛА ФОРМИРУЕМЫЙ АДМИНИСТРАТИВНО-ФИСКАЛЬНЫЙ АППАРАТ К СИСТЕМЕ ОБЛАСТНЫХ УЧРЕЖДЕНИЙ. ИДЕЯ ТАКОЙ СВЯЗИ НЕ ПРОПАЛА, КАК МИМОХОДОМ БРОШЕННОЕ СЛОВО, А ПОЛУЧИЛА БОЛЕЕ ДЕТАЛЬНУЮ ПРОРАБОТКУ ВСЕГО НЕСКОЛЬКО МЕСЯЦЕВ СПУСТЯ, КОГДА НА СВЕТ ПОЯВИЛСЯ ЦЕЛЫЙ ПАКЕТ УКАЗОВ-ИНСТРУКЦИЙ, ОПРЕДЕЛИВШИЙ КРУГ ДОЛЖНОСТНЫХ ОБЯЗАННОСТЕЙ И ПОЛНОМОЧИЙ ЧИНОВ ОБЛАСТНОГО УПРАВЛЕНИЯ. 
НАПИСАННЫЕ В СООТВЕТСТВИИ С ОБЩИМ ДУХОМ НАМЕЧАЕМОЙ РЕФОРМЫ (РАЗДЕЛЕНИЕ ПРЕДМЕТОВ ВЕДЕНИЯ МЕЖДУ РАЗЛИЧНЫМИ ЧИНОВНИКАМИ МЕСТНОЙ АДМИНИСТРАЦИИ), ИНСТРУКЦИИ 1719 Г. СУЩЕСТВЕННО ОТЛИЧАЛИСЬ ОТ НОЯБРЬСКИХ УКАЗОВ 1718 Г. В ЦЕЛОМ РЯДЕ ДЕТАЛЕЙ (ЗАМЕТНОЙ РЕВИЗИИ ПОДВЕРГЛИСЬ ДАЖЕ ДОЛЖНОСТНЫЕ НАИМЕНОВАНИЯ МЕСТНЫХ НАЧАЛЬНИКОВ), НО САМА ИДЕЯ ВЗАИМОДЕЙСТВИЯ МЕЖДУ СБОРЩИКАМИ ПОДУШНОЙ ПОДАТИ И ДОЛЖНОСТНЫМИ ЛИЦАМИ ОБЛАСТИ ПРИОБРЕЛА БОЛЬШУЮ МАСШТАБНОСТЬ И ОПРЕДЕЛЕННОСТЬ. ОСОБЕННО ИНФОРМАТИВНОЙ В ЭТОМ ОТНОШЕНИИ ОКАЗАЛАСЬ « ИНСТРУКЦИЯ, ИЛИ НАКАЗ ВОЕВОДАМ», АДРЕСОВАННАЯ СТАРШИМ НАЧАЛЬНИКАМ МЕСТНОГО АППАРАТА ПРОВИНЦИАЛЬНЫМ ВОЕВОДАМ И, ПО УМОЛЧАНИЮ, ГУБЕРНАТОРАМ. ЧЕТЫРЕ ПУНКТА ЭТОЙ ИНСТРУКЦИИ ОПРЕДЕЛЯЛИ СХЕМУ ВЗАИМООТНОШЕНИЙ ВОЕВОД И ЛИЦ ВОЕННО-ПОДАТНОГО ВЕДОМСТВА. В ПЕРВУЮ ОЧЕРЕДЬ, ВОЕВОДЫ ДОЛЖНЫ БЫЛИ СОДЕЙСТВОВАТЬ ПРОЦЕДУРЕ РАСПОЛОЖЕНИЯ ВОИНСКОЙ ЧАСТИ НА ПОСТОЙ В ТОМ ИЛИ ИНОМ СЕЛЕ; ИМ ПОЛАГАЛОСЬ ИМЕТЬ « ИСТИННЫЕ РАСЧЕТЫ» О «ЗАСЛУЖЕННОМ СОЛДАТСКОМ ЖАЛОВАНЬЕ» (Т.Е. РАСПОЛАГАТЬ ТОЧНЫМИ СВЕДЕНИЯМИ О ТОМ, СКОЛЬКО ПОЛОЖЕНО СОБИРАТЬ СРЕДСТВ С КРЕСТЬЯН НА СОДЕРЖАНИЕ РАСКВАРТИРОВАННЫХ СОЛДАТ). ВОЕВОДА НАДЕЛЯЛСЯ ПРАВОМ СВОЕГО РОДА ТРЕТЕЙСКОГО СУДА МЕЖДУ ПОЛКОВЫМ КОМИССАРОМ И ОФИЦЕРАМИ ПОЛКА, С ОДНОЙ СТОРОНЫ, И ЗЕМСКИМ КОМИССАРОМ И КРЕСТЬЯНАМИ, С ДРУГОЙ: «В ОБИДАХ И В ССОРАХ И ВО ВСЯКИХ НЕПОРЯДКАХ ЧИНИТЬ РАССМОТРЕНИЕ, ЧТОБЫ НА ОБЕ СТОРОНЫ БЫЛО БЕЗОБИДНО...» ${ }^{10}$. АНАЛОГИЧНЫЕ ПОЛНОМОЧИЯ ДАВАЛИСЬ ВОЕВОДАМ И В ОТНОШЕНИИ СНАБЖЕНИЯ ФЛОТСКИХ КОМАНД : « А БУДЕ ЧЕГО НА ФЛОТ ТРЕБОВАТЬ БУДУТ, О ТОМ ОФИЦЕРАМ УКАЗЫ ПОСЫЛАТЬ И С СЕЛ СОБИРАТЬ, ЧТО ОНЫМ НА ПОХОД ВЫДОВАТЬ ДОЛЖНО». САМ СБОР ПОДУШНОЙ ПОДАТИ ТАКЖЕ ОКАЗЫВАЛСЯ ПОДВЕДОМСТВЕНЕН ВОЕВОДАМ ; ИНСТРУКЦИЯ УПОЛНОМАЧИВАЛА ИХ НАДЗИРАТЬ ЗА ДЕЯТЕЛЬНОСТЬЮ КОМИССАРОВ ОТ ЗЕМЛИ И ПРИДАННЫХ ИМ ПОДЬЯЧИХ, ЧТОБЫ ОНИ «ЧИНИЛИ СУЩУЮ ПРАВДУ» В СБОРАХ. НАКОНЕЦ, ВОЕВОДА БЫЛ ОБЯЗАН НАБЛЮДАТЬ ЗА ПРОВЕДЕНИЕМ САМОЙ ПОДУШНОЙ ПЕРЕПИСИ, ИМЕЯ ПРАВО ПРОВОДИТЬ РОЗЫСК И НАКАЗЫВАТЬ ВИНОВНЫХ В СЛУЧАЕ ВЫЯВЛЕНИЯ ЗЛОУПОТРЕБЛЕНИЙ. ВИДИМО, РЕАЛИЗУЯ ЭТО ПРАВО, ВОЕВОДА БЫЛ В ПОЛНОЙ ВЛАСТИ : О НАЛОЖЕННЫХ САНКЦИЯХ ЕМУ ПОЛАГАЛОСЬ ЛИШЬ УВЕДОМЛЯТЬ КАМЕР-КОЛЛЕГИЮ ${ }^{11}$. КАК ВИДИМ, ВОЕВОДЫ ПОЛУЧАЛИ ШИРОКИЙ КРУГ КОМПЕТЕНЦИЙ ПО АДМИНИСТРАТИВНОМУ НАДЗОРУ ЗА ВАЖНЕЙШИМИ ПРОЦЕДУРАМИ, СВЯЗАННЫМИ С РЕАЛИЗАЦИЕЙ ПОДАТНОЙ РЕФОРМЫ НА ВВЕРЕННЫХ ИМ ТЕРРИТОРИЯХ, И НАДЕЛЯЛИСЬ ВЛАСТЬЮ ДЛЯ ВМЕШАТЕЛЬСТВА В ДЕЯТЕЛЬНОСТЬ КОМИССАРОВ ОТ ЗЕМЛИ, ПЕРЕПИСЧИКОВ И (В МЕНЬШЕЙ СТЕПЕНИ) ПОЛКОВЫХ ПРЕДСТАВИТЕЛЕЙ. ЭТО ОЗНАЧАЛО, ЧТО ПЕТР НЕ ТОЛЬКО НЕ ОТКАЗАЛСЯ ОТ МЫСЛИ ПРИВЕСТИ В ЕДИНУЮ СИСТЕМУ ОРГАНИЗАЦИЮ МЕСТНОГО УПРАВЛЕНИЯ, ФИСКА И СОДЕРЖАНИЯ ВОЙСК, НО СУЩЕСТВЕННО РАЗВИЛ И НОРМАТИВНО УКРЕПИЛ ЕЕ.

ОДНАКО ПРИ ВСЕЙ ДЕТАЛИЗАЦИИ ПОЛНОМОЧИЙ НОВОГО ОБЛАСТНОГО АППАРАТА, ЗАКОНОДАТЕЛЬСТВО 1719 Г. ПРОДОЛЖАЛО ИЗОБИЛОВАТЬ НЕДОГОВОРЕННОСТЯМИ, УМОЛЧАНИЯМИ И ОТКРОВЕННЫМИ ПРОТИВОРЕЧИЯМИ, ПОЗВОЛЯВШИМИ ПРОИЗВОЛЬНЫЕ ТОЛКОВАНИЯ И НЕИЗБЕЖНО ПОРОЖДАВШИМИ НАПРЯЖЕННОСТЬ ВО ВЗАИМООТНОШЕНИЯХ УПРАВЛЕНЦЕВ РАЗНОЙ ВЕДОМСТВЕННОЙ ПОДЧИНЕННОСТИ. ЭТО НЕ ТОЛЬКО СОЗДАВАЛО ЗАТРУДНЕНИЯ В РЕАЛИЗАЦИИ ПРАВ ВОЕВОД, НО СТАВИЛО В ДВУСМЫСЛЕННОЕ ПОЛОЖЕНИЕ ДРУГИХ ОБЛАСТНЫХ ЧИНОВНИКОВ, В ПЕРВУЮ ОЧЕРЕДЬ ТЕХ, ЧЬИ ДОЛЖНОСТНЫЕ ОБЯЗАННОСТИ ПОЛНОСТЬЮ ИЛИ ПО ПРЕИМУЩЕСТВУ ЛЕЖАЛИ В 
ФИНАНСОВО-ЭКОНОМИЧЕСКОЙ СФЕРЕ : ЗЕМСКИХ КАМЕРИРОВ (ЗЕМСКИХ НАДЗИРАТЕЛЕЙ СБОРОВ) И ЗЕМСКИХ КОМИССАРОВ (РУКОВОДИТЕЛЕЙ НИЗШЕГО ЗВЕНА МЕСТНОГО УПРАВЛЕНИЯ), В ДАННЫХ ИМ ИНСТРУКЦИЯХ САМЫМ ТЩАТЕЛЬНЫМ ОБРАЗОМ ПЕРЕЧИСЛЯЛИСЬ ИХ ФУНКЦИИ ПО ОБЕСПЕЧЕНИЮ СБОРОВ И ОФОРМЛЕНИЮ ФИНАНСОВОЙ ОТЧЕТНОСТИ, НО ИХ ОТНОШЕНИЕ К РЕАЛИЗАЦИИ ПОДАТНОЙ РЕФОРМЫ ОПРЕДЕЛЯЛОСЬ КРАЙНЕ НЕДОСТАТОЧНО: «ЗЕМСКОГО КОМИССАРА ГЛАВНЕЙШАЯ ДОЛЖНОСТЬ В ТОМ СОСТОИТ, ЧТОБ ОН В ПОВЕРЕННОМ ЕМУ УЕЗДЕ В НАДЛЕЖАЩЕЕ И УКАЗНОЕ ВРЕМЯ ВСЕ... ДОХОДЫ СОБИРАЛ И ПРИПИСЫВАЛ, КРОМЕ ПОШЛИН...», - НЕДВУСМЫСЛЕНО ЗАЯВЛЯЛА «ИНСТРУКЦИЯ, ИЛИ НАКАЗ ЗЕМСКИМ КОМИССАРАМ ${ }^{12}$. «ЗЕМСКОМУ НАДЗИРАТЕЛЮ СБОРОВ ВСЕ ДОХОДЫ, ПРИХОДЫ И РАСХОДЫ И ВЕДОМОСТИ О СБОРАХ, ПОДАННЫЕ ОТ ЗЕМСКИХ КОМИССАРОВ, ИСТИННО ЗАПИСЫВАТЬ И СО ВСЕХ ИМЕТЬ ПРАВДИВУЮ ЗАПИСНУЮ КНИГУ С АЛФАВИТНЫМ РЕГИСТРОМ», - НЕ МЕНЕЕ ОПРЕДЕЛЕННО ГЛАСИЛ П. 41 « ИНСТРУКЦИИ, ИЛИ НАКАЗА ВОЕВОДАМ», ПОВЕСТВОВАВШИЙ О ПОМОЩНИКАХ ВОЕВОДЫ ПО ФИНАНСОВОЙ ЧАСТИ ${ }^{13}$. ЗЕМСКИЙ КАМЕРИР ВЕДЕТ ПЕРЕПИСНУЮ КНИГУ « ВСЕМ ДЕРЕВНЯМ, ДВОРАМ И В НИХ ЛЮДЯМ, ТАКО Ж ЗЕМЛЯМ И НАДЛЕЖАЩИМ УГОДЬЯМ, С КОТОРЫХ ГОСУДАРЕВЫ ПОДАТИ БЕРУТСЯ» ; ЭТА КНИГА - « ГЛАВНЕЙШЕЕ ОСНОВАНИЕ», НА КОТОРОМ «ВСЕ КОМИССАРСКИЕ СЧЕТА И ВСЕ ПРЕДЫДУЩИЕ (Т.Е. «ВПРЕДЬ ИДУЩИЕ», БУДУЩИЕ. - Д.Р.) ОКЛАДНЫЕ И НЕОКЛАДНЫЕ СБОРЫ И ПОДАТИ ВПРЕДЬ ОСНОВЫВАТЬСЯ ИМЕЮТ», - УТОЧНЯЛА « ИНСТРУКЦИЯ ЗЕМСКИМ КАМЕРИРАМ» ${ }^{14}$.

КАК СЛЕДОВАЛО ПОНИМАТЬ ВСЕ ЭТИ НОРМЫ ? ПРЕДПОЛАГАЛ ЛИ ЗАКОНОДАТЕЛЬ, ЧТО ПОД ВСЕМИ «ДОХОДАМИ И СБОРАМИ», НАХОДИВШИМИСЯ В ВЕДЕНИИ КАМЕРНЫХ ЧИНОВНИКОВ, СКРЫВАЛАСЬ И ПОДУШНАЯ ПОДАТЬ ? ВОПРОС ЭТОТ БЫЛ ОСТАВЛЕН БЕЗ ОТВЕТА, И ЕГО ТОЛКОВАНИЕ, ОЧЕВИДНО, ОТДАВАЛОСЬ НА ВОЛЮ СЛУЧАЯ ИЛИ БУДУЩИХ УТОЧНЯЮЩИХ НОРМАТИВОВ. УПОТРЕБЛЕНИЕ В ЦИТИРОВАННЫХ ПУНКТАХ ИНСТРУКЦИЙ СТАРЫХ ПОНЯТИЙ ОКЛАДНЫХ И НЕОКЛАДНЫХ СБОРОВ, ДЕРЕВЕНЬ И ДВОРОВ КАК НАЛОГООБЛАГАЕМЫХ ЕДИНИЦ КАК БУДТО СВИДЕТЕЛЬСТВУЕТ О ТОМ, ЧТО ПРИ НАПИСАНИИ ЭТИХ ДОКУМЕНТОВ ПЕТР УПУСТИЛ ИЗ ВИДУ ИЗМЕНЕНИЯ В СИСТЕМЕ НАЛОГООБЛОЖЕНИЯ И УЧЕТА ПОДАТНОГО НАСЕЛЕНИЯ. ДАЖЕ В ТЕХ ПУНКТАХ « ИНСТРУКЦИИ, ИЛИ НАКАЗА ЗЕМСКИМ КОМИССАРАМ», ГДЕ РЕЧЬ ИДЕТ О ПЕРЕПИСИ И ДВИЖЕНИИ ВОЙСК, НОВЫЕ РЕАЛИИ ОТСУТСТВУЮТ. ЗЕМСКИЙ КОМИССАР СО СВОИМИ ПОДЧИНЕННЫМИ СЛУЖИТЕЛЯМИ, НАПРИМЕР, ОБЯЗАН ПРИСУТСТВОВАТЬ ПРИ « ПЕРЕПИСКЕ ДВОРОВ ИЛИ ЗЕМЕЛЬ» СВОЕГО УЕЗДА, ЕСЛИ ТАКОВАЯ СЛУЧИТСЯ, ЧТОБЫ ОКАЗЫВАТЬ СОДЕЙСТВИЕ «УЧРЕЖДЕННЫМ ПЕРЕПИСЧИКАМ»; ЕСЛИ ЧЕРЕЗ УЕЗД СЛУЧИТСЯ « ВОЙСКУ ПРОХОД ИЛИ ОНЫЕ ОТ НАЧАЛЬСТВА В УЕЗДЕ ПОСТАВЛЕНЫ БУДУТ», ТО ЗЕМСКИЙ КОМИССАР ДОЛЖЕН БЫЛ СНАБЖАТЬ «ВОИНСКИХ ЛЮДЕЙ» ВСЕМ НЕОБХОДИМЫМ И СМОТРЕТЬ, ЧТОБЫ ТЯГОТЫ ПО ОБЕСПЕЧЕНИЮ ВОЙСК РАВНОМЕРНО РАСПРЕДЕЛЯЛИСЬ МЕЖДУ ВСЕМИ ТЯГЛЕЦАМИ ${ }^{15}$. ТАКИЕ НЕСООБРАЗНОСТИ ОСТАЕТСЯ ТОЛЬКО ОТНЕСТИ НА СЧЕТ НЕСОВЕРШЕНСТВА ЮРИДИЧЕСКОЙ ТЕХНИКИ И ПРЕДПОЛОЖИТЬ, ЧТО ПЕТР, В СВОЙСТВЕННОЙ ЕМУ МАНЕРЕ ОПРЕДЕЛИВ ВЗАИМООТНОШЕНИЯ СТАРШЕГО ЧИНОВНИКА ОБЛАСТНОГО УПРАВЛЕНИЯ (ВОЕВОДЫ) С ПЕРЕПИСЧИКАМИ И СБОРЩИКАМИ ПОДУШНОЙ ПОДАТИ, ДЕЙСТВИТЕЛЬНО ОСТАВИЛ НА ПОТОМ ДЕТАЛЬНУЮ ПРОРАБОТКУ ОСТАЛЬНЫХ НОРМ. ЦАРЬ, НЕСОМНЕННО, ТОРОПИЛСЯ : ПРОВЕДЕНИЕ ПОДУШНОЙ ПЕРЕПИСИ ВИДЕЛОСЬ ЕМУ ДЕЛОМ ОДНОГО ГОДА, НЕОБХОДИМО БЫЛО СРОЧНО РАЗМЕЩАТЬ ВОЙСКА ПО ЗИМНИМ КВАРТИРАМ, И ОН НАХОДИЛ ВПОЛНЕ ЕСТЕСТВЕННЫМ ПОРУЧИТЬ ЭТИ ПРОЦЕДУРЫ МЕСТНЫМ АДМИНИСТРАЦИЯМ. ДРУГОГО ВЫХОДА, КАК КАЗАЛОСЬ, У НЕГО ПРОСТО НЕ БЫЛО. 
ИХ ВИНЫ. БЕСКОНЕЧНЫЕ ПРЕОБРАЗОВАНИЯ, КАДРОВЫЕ ПЕРЕТРЯСКИ, ПРОТИВОРЕЧИВЫЕ УКАЗАНИЯ, КАТАСТРОФИЧЕСКИ НАРАСТАВШИЙ ВАЛ ОТЧЕТНОСТИ, МАЛОЧИСЛЕННЫЕ И ПЛОХО ПОНИМАВШИЕ ПРОИСХОДЯЩЕЕ КАНЦЕЛЯРИСТЫ, КОТОРЫХ К ТОМУ ЖЕ ТО И ДЕЛО ЗАБИРАЛИ НА РАБОТУ В ПОСТОЯННО СОЗДАВАЕМЫЕ КОМИССИИ И СТРУКТУРЫ СПЕЦИАЛЬНОГО УПРАВЛЕНИЯ - ВСЕ ЭТО БУКВАЛЬНО ПАРАЛИЗОВЫВАЛО ДЕЯТЕЛЬНОСТЬ ГОСУДАРСТВЕННОГО АППАРАТА НА МЕСТАХ. ОБОБЩЕНИЮ ДАННЫХ ПОДУШНОЙ ПЕРЕПИСИ, СТАЛА СПЕЦИАЛЬНО СОЗДАННАЯ КАНЦЕЛЯРИЯ ПЕРЕПИСНЫХ ДЕЛ. ОНА РАСПОЛАГАЛАСЬ В ПЕТЕРБУРГЕ, БЫЛА ПОДЧИНЕНА СЕНАТУ И РАБОТАЛА ПОД РУКОВОДСТВОМ ГЕНЕРАЛ-РЕВИЗОРА БРИГАДИРА В.Н. ЗОТОВА, ПОЧЕМУ НЕРЕДКО И НАЗЫВАЛАСЬ (ПО ТРАДИЦИИ ВРЕМЕНИ) КАНЦЕЛЯРИЕЙ ГЕНЕРАЛ-РЕВИЗОРА ЗОТОВА. ДАВАЛИСЬ ОЧЕНЬ ТРУДНО, И К КОНЦУ 1719 Г. ПЕРЕПИСЬ, РАЗУМЕЕТСЯ, БЫЛА ЕЩЕ ДАЛЕКА ОТ ЗАВЕРШЕНИЯ ${ }^{16}$. ГОД СПУСТЯ ВЫЯСНИЛОСЬ, ЧТО СОБРАННАЯ ИНФОРМАЦИЯ НЕПОЛНА И НЕДОСТОВЕРНА, И ОВНАРУЖИЛАСЬ ПОТРЕБНОСТЬ В ЕЕ ПРОВЕРКЕ ПУТЕМ РЕВИЗИИ. ПЕРВОНАЧАЛЬНО ПЕТР ПОПЫТАЛСЯ ВЫНУДИТЬ САМИХ ДУШЕВЛАДЕЛЬЦЕВ ПОДАТЬ УТОЧНЕННЫЕ И ДОПОЛНЕННЫЕ СВЕДЕНИЯ ${ }^{17}$, УГРОЖАЯ ОСЛУШНИКАМ КОНФИСКАЦИЕЙ ИМЕНИЙ ${ }^{18}$. ПАРАЛЛЕЛЬНО ОН ПРЕДПРИНЯЛ МЕРЫ ДЛЯ АКТИВИЗАЦИИ ДЕЯТЕЛЬНОСТИ МЕСТНЫХ АДМИНИСТРАЦИЙ. СРЕДСТВО НАШЛОСЬ ОПРОБОВАННОЕ ГВАРДЕЙЦЫ-ПОНУДИТЕЛИ, КОТОРЫЕ И РАНЬШЕ ИСПОЛЬЗОВАЛИСЬ В ДЕЛАХ ТАКОГО РОДА 19. СНАБЖЕННЫЕ ИМЕННЫМИ ИНСТРУКЦИЯМИ ГОСУДАРЯ, ОНИ ОБЛАДАЛИ ПРАВОМ НАЛАГАТЬ НА МЕСТНЫХ ЧИНОВНИКОВ ЛЮБОГО РАНГА АДМИНИСТРАТИВНЫЙ АРЕСТ И ДЕРЖАТЬ ИХ В КАНДАЛАХ И НА ШЕЙНОЙ ЦЕПИ В КАНЦЕЛЯРИЯХ ДО ТЕХ ПОР, ПОКА НЕ ДОБИВАЛИСЬ ИСПОЛНЕНИЯ ИСКОМОГО: СБОРА НЕДОИМОК ИЛИ ПРЕДСТАВЛЕНИЯ ТОЙ ИЛИ ИНОЙ ОТЧЕТНОЙ ДОКУМЕНТАЦИИ. ОДНАКО ЧРЕЗВЫЧАЙНЫЕ МЕРЫ НЕ ПРИНЕСЛИ ОЖИДАЕМОГО ЭФФЕКТА, И В ЯНВАРЕ 1721 Г. СЕНАТ ИЗДАЛ УКАЗ О ВЗЯТИИ ВОЕВОД К РОЗЫСКУ В ПЕТЕРБУРГ И О КОНФИСКАЦИИ ИХ НЕДВИЖИМОСТИ ${ }^{20}$. В КОНЦЕ КОНЦОВ, РАЗУВЕРИВШИСЬ В СПОСОБНОСТИ ГРАЖДАНСКИХ ВЛАСТЕЙ ОБЕСПЕЧИТЬ СТОЛЬ ВАЖНОЕ МЕРОПРИЯТИЕ, ПЕТР РЕШИЛ ПРИБЕГНУТЬ К СВОЕМУ ПОСЛЕДНЕМУ, САМОМУ ГЛАВНОМУ РЕЗЕРВУ - АРМИИ.

В ЯНВАРЕ 1722 Г. ВСЯ ДЕЯТЕЛЬНОСТЬ ПО РЕВИЗИИ ЧИСЛА МУЖСКОГО ТЯГЛОГО НАСЕЛЕНИЯ В СТРАНЕ ОКАЗАЛАСЬ ПЕРЕДАНА В РУКИ УПОЛНОМОЧЕННЫХ ГЕНЕРАЛОВ И ШТАБ-ОФИЦЕРОВ. ПРИ НИХ СФОРМИРОВАЛИСЬ ОСОБЫЕ СТРУКТУРЫ, УКОМПЛЕКТОВАННЫЕ ОФИЦЕРАМИ ПОЛКОВ, КОТОРЫЕ ДОЛЖНЫ БЫЛИ БЫТЬ РАСКВАРТИРОВАНЫ В ГУБЕРНИЯХ. ИМЕНУЕМЫЕ В ИСТОЧНИКАХ «ГЕНЕРАЛИТЕТОМ», ИЛИ «ПЕРЕПИСЧИКАМИ», ЭТИ СТРУКТУРЫ ПОЛУЧИЛИ В ЛИТЕРАТУРЕ ОБЩЕЕ НАИМЕНОВАНИЕ ПЕРЕПИСНЫХ КАНЦЕЛЯРИЙ ${ }^{21}$. ИЗ ТЕКСТА СЕНАТСКОГО УКАЗА, ДАННОГО В ВОЕННУЮ КОЛЛЕГИЮ 26 ЯНВАРЯ 1722 Г., ИЗВЕСТНЫ ИМЕНА ГЛАВ ЭТИХ КАНЦЕЛЯРИЙ : « В САНКТ-ПИТЕРБУРХСКОЙ - ГЕНЕРАЛ-МАЭОР ЧЕРНЫШОВ ; В МОСКОВСКОЙ - ГЕНЕРАЛЫ-МАЭОРЫ ВОЛКОВ, ЮСУПОВ ПОПОЛАМ ; В КИЕВСКОЙ - БРЕГАДИР ЛЕВАШОВ * ; В АЗОВСКОЙ - ПОЛКОВНИК МЯКИНИН ; В РИЖСКОЙ - ПОЛКОВНИК ВЕЛЬЯМИНОВ; В СИБИРСКОЙ - ПОЛКОВНИК СОНЦОВ ; В КАЗАНСКОЙ - БРЕГАДИР ФАМЕНГДИН ; В НИЖЕГОРОЦКОЙ - ГЕНЕРАЛ-МАЭОР САЛТЫКОВ ; В АСТРАХАНСКОЙ - БРЕГАДИР ФАМЕНГДИН ${ }^{22}$. 
ДЕЯТЕЛЬНОСТЬ ПЕРЕПИСНЫХ КАНЦЕЛЯРИЙ ПОДРОБНО ОПИСАНА М.М. БОГОСЛОВСКИМ И СУЩЕСТВЕННО ДОПОЛНЕНА ВАЖНЫМИ МАТЕРИАЛАМИ, ВЫЯВЛЕННЫМИ И ПРОАНАЛИЗИРОВАННЫМИ Е.В. АНИСИМОВЫМ ${ }^{23}$. ЭТО ИЗБАВЛЯЕТ НАС ОТ НЕОБХОДИМОСТИ ПОДРОБНО ОСТАНАВЛИВАТЬСЯ НА ДАННОМ СЮЖЕТЕ И ПОЗВОЛЯЕТ ЛИШЬ В ОБЩИХ ЧЕРТАХ ОБОЗНАЧИТЬ РОЛЬ, КОТОРУЮ ЭТИ КАНЦЕЛЯРИИ СЫГРАЛИ В ПРОЦЕССЕ ФОРМИРОВАНИЯ СИСТЕМЫ ВОЕННЫХ ДИСТРИКТОВ И УЧРЕЖДЕНИЙ ВОЕННО-ПОДАТНОГО УПРАВЛЕНИЯ. ПРАКТИЧЕСКИ С САМОГО НАЧАЛА СВОЕГО ВОЗНИКНОВЕНИЯ, ПЕРЕПИСНЫЕ КАНЦЕЛЯРИИ, ПОМИМО ГЛАВНОЙ ЗАДАЧИ (ОСУЩЕСТВЛЕНИЯ ПРОВЕРКИ ДАННЫХ ПЕРЕПИСИ, А ПО СУТИ - ПРОДОЛЖЕНИЯ ЕЕ ПРОВЕДЕНИЯ), ВЗЯЛИ НА СЕБЯ ОБЯЗАННОСТИ ПО РАЗМЕЩЕНИЮ ВОЙСК НА ЗИМНИХ КВАРТИРАХ И ОРГАНИЗАЦИИ АППАРАТА ВЗИМАНИЯ ПОДУШНОГО НАЛОГА, В ПЕРВУЮ ОЧЕРЕДЬ - ПРОВЕДЕНИЕ УЕЗДНЫХ СЪЕЗДОВ ПО ВЫБОРУ КОМИССАРОВ ОТ ЗЕМЛИ.

ПРОЦЕДУРА РОСПИСИ ПОЛКОВ НА ПОСТОЙ ПО ГУБЕРНИЯМ ОКАЗАЛА ОЧЕНЬ СЕРЬЕЗНОЕ ВЛИЯНИЕ НА АДМИНИСТРАТИВНО-ТЕРРИТОРИАЛЬНОЕ ДЕЛЕНИЕ СТРАНЫ. ДЕЛО В ТОМ, ЧТО ОКРУГ, НА ТЕРРИТОРИИ КОТОРОГО РАСПОЛАГАЛСЯ ПОЛК И НАСЕЛЕНИЕ КОТОРОГО ОБЕСПЕЧИВАЛО СОДЕРЖАНИЕ ПОЛКА ПУТЕМ ВЫПЛАТЫ ПОДУШНОЙ ПОДАТИ, ОБРАЗОВАЛ ОСОБУЮ АДМИНИСТРАТИВНО-ФИСКАЛЬНУЮ ТЕРРИТОРИАЛЬНУЮ ЕДИНИЦУ - ПОЛКОВОЙ ДИСТРИКТ. СЕТЬ ПОЛКОВЫХ ДИСТРИКТОВ, ТАКИМ ОБРАЗОМ, НАЛОЖИЛАСЬ НА АДМИНИСТРАТИВНО-ТЕРРИТОРИАЛЬНУЮ СЕТЬ, ОБРАЗОВАННУЮ ПРОВИНЦИЯМИ И ДИСТРИКТАМИ ОБЩЕГО ВЕДЕНИЯ, ВОЗНИКШИМИ В ХОДЕ ВТОРОЙ ОБЛАСТНОЙ РЕФОРМЫ. ИМЕННО В РАМКАХ ПОЛКОВЫХ ДИСТРИКТОВ, ЧЬИ ГРАНИЦЫ, КАК ПРАВИЛО, НЕ СОВПАДАЛИ С ГРАНИЦАМИ СУЩЕСТВОВАВШЕГО ТЕРРИТОРИАЛЬНОГО УСТРОЙСТВА, ДОЛЖНЫ БЫЛИ ДЕЙСТВОВАТЬ ВОЕННО-ПОДАТНЫЕ ОРГАНЫ, ОТВЕТСТВЕННЫЕ ЗА СБОР ПОДУШНОЙ ПОДАТИ. ПО УСТОЯВШЕЙСЯ ИСТОРИОГРАФИЧЕСКОЙ ТРАДИЦИИ, ЭТИ ОРГАНЫ ПРИНЯТО НАЗЫВАТЬ ПОЛКОВЫМИ ДВОРАМИ. ЭТА ТРАДИЦИЯ ЗАКРЕПЛЕНА СЕГОДНЯ НА УРОВНЕ УЧЕБНОЙ И СПРАВОЧНОЙ ЛИТЕРАТУРЫ. НАПРИМЕР, МАРИНА ВИЛЕНОВНА БАБИЧ, АВТОР СООТВЕТСТВУЮЩЕЙ СТАТЬИ В СОВРЕМЕННОМ СЛОВАРЕ-СПРАВОЧНИКЕ «ГОСУДАРСТВЕННОСТЬ РОССИИ», ОПРЕДЕЛЯЕТ ПОЛКОВОЙ ДВОР КАК « МЕСТНОЕ УЧРЕЖДЕНИЕ ВОЕННОГО УПРАВЛЕНИЯ ${ }^{24}$. РАЗВИВАЯ СВОЮ МЫСЛЬ, ИСТОРИК УТВЕРЖДАЕТ, ЧТО «ВСЕ ПОЛКОВЫЕ ДВОРЫ БЫЛИ, ВЕРОЯТНО, ОРГАНИЗОВАНЫ КАК ОСОБЫЕ ОТНОСИТЕЛЬНО ПОЛКОВЫХ КАНЦЕЛЯРИЙ ВЛАСТНЫЕ ИНСТИТУТЫ». С ЭТИМ НАБЛЮДЕНИЕМ СЛЕДУЕТ СОГЛАСИТЬСЯ ЛИШЬ ОТЧАСТИ : ПОЛКОВАЯ КАНЦЕЛЯРИЯ КАК УЧРЕЖДЕНИЕ, СОСРЕДОТОЧИВАВШЕЕ В СЕБЕ ДЕЛОПРОИЗВОДСТВО ПО ОБЩЕМУ УПРАВЛЕНИЮ, СТРОЕВОЙ И ХОЗЯЙСТВЕННОЙ ЧАСТИ ПОЛКА ${ }^{25}$, ДЕЙСТВИТЕЛЬНО СУЩЕСТВОВАЛА И ДО, И ПОСЛЕ ОРГАНИЗАЦИИ ПОЛКОВЫХ ДВОРОВ, ОТЛИЧАЯСЬ ДОСТАТОЧНО ЯСНОЙ КОМПЕТЕНЦИЕЙ. НО ВОТ ЯВЛЯЛСЯ ЛИ « УЧРЕЖДЕНИЕМ» ПОЛКОВОЙ двоР ? ЧТОБЫ ПОНЯТЬ СТРУКТУРУ И ДОЛЖНОСТНУЮ КОМПЕТЕНЦИЮ ПОЛКОВЫХ ДВОРОВ КАК УЧРЕЖДЕНИЙ, СЛЕДУЕТ ОБРАТИТЬСЯ К ЗАКОНОДАТЕЛЬСТВУ, В ПЕРВУЮ ОЧЕРЕДЬ К ТЕМ ТРЕМ, ХОРОШО ИЗВЕСТНЫМ В ИСТОРИОГРАФИИ, НОРМАТИВНО-ЗАКОНОДАТЕЛЬНЫМ АКТАМ, КОТОРЫЕ БЫЛИ ИЗДАНЫ 26 ИЮНЯ 1724 Г.: Т.Н. «ПЛАКАТУ», « ИНСТРУКЦИИ ПОЛКОВНИКУ» И « ИНСТРУКЦИИ ЗЕМСКОМУ КОМИССАРУ», А ТАКЖЕ ПРИМЫКАЮЩЕМУ К НИМ ИМЕННОМУ УКАЗУ «О ДОЛЖНОСТИ ПОЛКОВНИКА ПО НАБЛЮДЕНИЮ ЗЕМСКОЙ полиции». 
В ДВУХ ИЗ НАЗВАННЫХ ДОКУМЕНТОВ, « ИНСТРУКЦИИ ЗЕМСКОМУ КОМИССАРУ» И УКАЗЕ «О ДОЛЖНОСТИ ПОЛКОВНИКОВ», ПОЛКОВЫЕ ДВОРЫ НЕ УПОМИНАЮТСЯ ВОВСЕ. ВОЗМОЖНО, ЭТОМУ НЕ СЛЕДУЕТ УДИВЛЯТЬСЯ, УЧИТЫВАЯ, ЧТО ДАННЫЕ АКТЫ ЯВЛЯЮТСЯ ВТОРИЧНЫМИ ПО ОТНОШЕНИЮ К « ПЛАКАТУ» И « ИНСТРУКЦИИ ПОЛКОВНИКУ», ПОЧТИ ДОСЛОВНО ПОВТОРЯЯ ИЛИ НЕСКОЛЬКО УТОЧНЯЯ ПОЛОЖЕНИЯ ПОСЛЕДНИХ В ЧАСТЯХ, КАСАЮЩИХСЯ ПОЛНОМОЧИЙ ЗЕМСКОГО КОМИССАРА (КОМИССАРА ОТ ЗЕМЛИ) И АДМИНИСТРАТИВНО-ПОЛИЦЕЙСКИХ ФУНКЦИЙ ПОЛКОВНИКА. В «ПЛАКАТЕ» И « ИНСТРУКЦИИ ПОЛКОВНИКУ» ИНФОРМАЦИЯ О ПОЛКОВЫХ ДВОРАХ ИМЕЕТСЯ, ХОТЯ ОНА И НЕ СЛИШКОМ ОБИЛЬНА. ПОЛКОВОЙ ДВОР ТРИЖДЫ ФИГУРИРУЕТ В ПЕРВОМ ДОКУМЕНТЕ И ТРИЖДЫ - ВО ВТОРОМ. САМИ ПО СЕБЕ, ЭТИ СКРОМНЫЕ ЦИФРЫ ТОЖЕ, ВОЗМОЖНО, НЕ ПОКАЗАТЕЛЬНЫ ; ГОРАЗДО БОЛЬШЕЕ ЗНАЧЕНИЕ ДЛЯ НАШИХ ИЗЫСКАНИЙ ПРЕДСТАВЛЯЕТ ИЗ СЕБЯ КОНТЕКСТ ЭТИХ УПОМИНАНИЙ.

ВЕРЫЙ РАЗ ПОНЯТИЕ « ПОЛКОВОЙ ДВОР» ВСТРЕЧАЕТСЯ В П. 4 ЧАСТИ 1 « ПЛАКАТА» («О ЗЕМСКОМ КОМИССАРЕ»), ГДЕ РЕЧЬ ИДЕТ ОБ ОРГАНИЗАЦИИ СЪЕЗДА МЕСТНОГО ДВОРЯНСТВА ИЛИ МЕСТНЫХ « ОБЫВАТЕЛЕЙ» (В РАЙОНАХ, ГДЕ ДВОРЯНСТВА НЕ БЫЛО) ДЛЯ ВЫБОРОВ ЗЕМСКОГО КОМИССАРА. В СООТВЕТСТВИИ С НОРМАТИВОМ, ВЫБОРЩИКАМ СЛЕДОВАЛО СЪЕЗЖАТЬСЯ НА ПОЛКОВОЙ ДВОР, КОТОРЫЙ, Т.О., УСТАНАВЛИВАЛСЯ В КАЧЕСТВЕ МЕСТА ПРОВЕДЕНИЯ ПРОЦЕДУРЫ ВЫБОРОВ. ДВА СЛЕДУЮЩИХ УПОМИНАНИЯ ПОЛКОВОГО ДВОРА МЫ ОБНАРУЖИВАЕМ В П. 19 ЧАСТИ 2 « ПЛАКАТА» (« О ПОЛКОВНИКЕ С ОФИЦЕРЫ»). ЗДЕСЬ СОДЕРЖАТСЯ ОБЩИЕ УКАЗАНИЯ О СТРОИТЕЛЬСТВЕ ЗИМНИХ КВАРТИР, В ТОМ ЧИСЛЕ О ТОМ, ЧЬИМИ СИЛАМИ СЛЕДУЕТ ОСУЩЕСТВЛЯТЬ НАЗВАННЫЕ РАБОТЫ. ПРОЦИТИРУЕМ ЭТОТ ФРАГМЕНТ :

ТАКЖЕ В СРЕДИНЕ ПОЛКА СДЕЛАТЬ ШТАБУ ДВОР ВОСЕМЬ ИЗБ И ПРИ ТОМ ДВОРЕ ШПИТАЛЬ И САРАИ НА ТЕЛЕГИ И ЯЩИКИ ПОЛКОВЫЕ <...> А СТРОИТЬ ТЕ СЛОБОДЫ И ДВОРЫ ПОЛКОВОЙ И ШТАБНОЙ И ПРОЧЕЕ ПОЛКАМИ [Т.Е. СИЛАМИ ЛИЧНОГО СОСТАВА ПОЛКА. - Д.Р.] <... А КОТОРЫЕ ПОЛКИ БУДУТ ПОСЕЛЕНЫ ОКОЛО САНКТ-ПЕТЕРБУРГА И В ДРУГИХ ТАМОШНИХ МЕСТАХ <... СЛОБОД НЕ СТРОИТЬ ; СДЕЛАТЬ ТОКМО ДЛЯ ЖИТЬЯ ОФИЦЕРАМ И КОМИССАРУ ЗЕМСКОМУ ПОЛКОВЫЕ ДВОРЫ КРЕСТЬЯНАМИ» ${ }^{26}$.

ТРИ УПОМИНАНИЯ ПОЛКОВЫХ ДВОРОВ, СОДЕРЖАЩИЕСЯ В « ИНСТРУКЦИИ ПОЛКОВНИКУ», СКОНЦЕНТРИРОВАНЫ В ПРОСТРАННОМ П. 12 («КОГДА ПОЛК ОТ КВАРТИР БУДЕТ В ОТЛУЧЕНИИ, КОГО ОСТАВИТЬ В КВАРТИРАХ И КАК ИМ ПОСТУПАТЬ»). ОФИЦЕРАМ, ОСТАЮЩИМСЯ НА ЗИМНИХ КВАРТИРАХ, ПОЛАГАЛОСЬ ИМЕТЬ В ТОМ МЕСТЕ КВАРТИРУ ВКУПЕ С КОМИССАРАМИ ОТ ЗЕМЛИ НА ПОЛКОВОМ ДВОРЕ» (П. 12.1); ИМ ЖЕ НАДЛЕЖАЛО « ПОЛКОВОЙ ДВОР ТОГО ПОЛКУ ИМЕТЬ <...> В ПРИЛЕЖНОМ СМОТРЕНИИ, ДАБЫ БЫЛ ЦЕЛ ; И БУДЕ ЧТО ПОПОРТИТСЯ, ЧИНИТЬ ОСТАВШИМИ ПРИ ПОЛКУ СОЛДАТАМИ» (П. 12.3); НАКОНЕЦ, «КОГДА ПО ОКОНЧАНИИ ГОДА БУДЕТ ИМ ПЕРЕМЕНА, ТОГДА ИМ, ОФИЦЕРАМ, ВСЮ СВОЮ КОМИССИЮ <... О ОТДАТЬ ; А ПОЛКОВОЙ ДВОР СДАТЬ ПО ОПИСИ ТЕМ ОФИЦЕРАМ, КОТОРЫЕ НА ПЕРЕМЕНУ ИМ <... ПРИСЛАНЫ БУДУТ» $(\text { П. 12.5 })^{27}$.

ТАКИМ ОБРАЗОМ, ВСЕ ШЕСТЬ УПОМИНАНИЙ ПОЛКОВЫХ ДВОРОВ В НОРМАТИВНЫХ ДОКУМЕНТАХ, ОПРЕДЕЛЯВШИХ БАЗОВЫЕ ПРАВА И ОБЯЗАННОСТИ ВОЕННО-ФИСКАЛЬНОЙ АДМИНИСТРАЦИИ, КРАСНОРЕЧИВО ГОВОРЯТ О ТОМ, ЧТО ПОЛКОВОЙ ДВОР ПОНИМАЛСЯ ЗАКОНОДАТЕЛЕМ ИСКЛЮЧИТЕЛЬНО КАК МЕСТО, КАК ЖИЛОЙ И ХОЗЯЙСТВЕННЫЙ КОМПЛЕКС, ГДЕ ПРОЖИВАЛИ И ДЕЙСТВОВАЛИ ТЕ ИЛИ ИНЫЕ ЛЮДИ ; КАК ФИЗИЧЕСКИЙ ОБЪЕКТ, КОТОРЫЙ МОГ ВЕТШАТЬ И ЛОМАТЬСЯ И КОТОРЫЙ НАДО БЫЛО ПОДДЕРЖИВАТЬ В 
ПРИГОДНОМ СОСТОЯНИИ. ВСЕ ЭТО НЕ ДАЕТ НАМ ОСНОВАНИЯ ВИДЕТЬ В ПОЛКОВОМ ДВОРЕ АДМИНИСТРАТИВНОЕ УЧРЕЖДЕНИЕ. 1724-1735 ГГ., ЕСТЬ ОКОЛО ДВУХ ДЕСЯТКОВ ДОКУМЕНТОВ, В КОТОРЫХ ПОЛНОСТЬЮ ВОСПРОИЗВЕДЕНЫ НАИМЕНОВАНИЯ УЧРЕЖДЕНИЙ-КОНТРАГЕНТОВ. ДВА ДЕСЯТКА ПРИМЕРОВ - НЕ СЛИШКОМ ВНУШИТЕЛЬНАЯ ВЫБОРКА ИЗ ВСЕГО МАССИВА СОХРАНИВШИХСЯ ДЕЛ, НО И ОНИ СПОСОБНЫ ДАТЬ ПИЩУ ДЛЯ РАЗМЫШЛЕНИЙ. ПЕРВОЕ, ЧТО БРОСАЕТСЯ В ГЛАЗА, - РЕДКОСТЬ УПОМИНАНИЙ ПОЛКОВЫХ ДВОРОВ В КАЧЕСТВЕ СУБЪЕКТОВ ПЕРЕПИСКИ, ПРИЧЕМ ОДНОЗНАЧНО ИСТОЛКОВАТЬ ИНСТАНЦИОННЫЙ СТАТУС ЭТИХ ДВОРОВ НЕ ПРЕДСТАВЛЯЕТСЯ ВОЗМОЖНЫМ. ТАК, В 1731 Г., БЫЛА ПОСЛАНА В ВЕРХОТУРСКУЮ ВОЕВОДСКУЮ КАНЦЕЛЯРИЮ ПРОМЕМОРИЯ « НОВГОРОЦКОГО АРМЕЙСКОГО ПЕХОТНОГО ПОЛКУ ИС ПОЛКОВОГО ДВОРА ЗБОРУ ПОДУШНЫХ ДЕНЕГ ЗА РУКОЮ КАПИТАНА КАЗАКОВА» ${ }^{28}$. В ТОМ ЖЕ 1731 Г. НА «НА ПОЛКОВОЙ ДВОР К ПОДУШНОМУ ЗБОРУ» В ТЮМЕНИ ПОСТУПИЛО ДОНОШЕНИЕ, А ПО СУТИ - ИЗВЕТ, В КОТОРОМ ДОНОСИТЕЛЬ ОБВИНЯЛ ОДНОГО ИЗ ПОДЬЯЧИХ ТЮМЕНСКОЙ ВОЕВОДСКОЙ КАНЦЕЛЯРИИ В УКЛОНЕНИИ ОТ УПЛАТЫ ПОДУШНОЙ ЗА КАБАЛЬНОГО ЧЕЛОВЕКА ${ }^{29}$. «СИБИРСКОГО ГВАРНИЗОНА ТОБОЛЬСКОГО ПЕХОТНОГО ПОЛКУ В ПОЛКОВОЙ ДВОР К ПОДУШНОМУ ЗБОРУ» ПРИШЛА ПРОМЕМОРИЯ «ИС ТЮМЕНСКОЙ КАНЦЕЛЯРИИ ВОЕВОЦКОГО ПРАВЛЕНИЯ» $(1731 \text { Г. })^{30}$. И, НАКОНЕЦ, СОВСЕМ ИНТЕРЕСНОЕ : « ОРЛОВСКОЙ ПРАВИНЦЫИ В КАНЦЕЛЯРИЮ СМОТРЕНИЯ НАД ПОДУШНЫМ ЗБОРОМ ГОРОДА НОВОСИЛЯ ИС ПОЛКОВОГО ДВОРА ДОНОШЕНИЕ» $(1735 \text { Г.) })^{31}$. В ПРИВЕДЕННЫХ СЛУЧАЯХ СМУЩАЕТ ТО, ЧТО ПОНЯТИЕ « ПОЛКОВОЙ ДВОР» КАК БЫ ДВОИТСЯ: ДОПОЛНЕНИЕ «К ПОДУШНОМУ СБОРУ» В КОНЦЕ КОНЦОВ ПРЕВРАЩАЕТСЯ В ПРОТИВОПОСТАВЛЕНИЕ (« ИЗ ПОЛКОВОГО ДВОРА» - «В КАНЦЕЛЯРИЮ СМОТРЕНИЯ НАД ПОДУШНЫМ СБОРОМ»).

СМУЩЕНИЕ ТОЛЬКО НАРАСТАЕТ, КОГДА МЫ НАЧИНАЕМ ЗНАКОМИТЬСЯ С ДРУГИМИ ДЕЛОПРОИЗВОДСТВЕННЫМИ ФОРМУЛИРОВКАМИ, КОТОРЫЕ ЯВНО ПРЕОБЛАДАЮТ И В 
КОТОРЫХ ПОЛКОВОЙ ДВОР «УХОДИТ В ТЕНЬ» И ВОВСЕ ИСЧЕЗАЕТ, УСТУПАЯ МЕСТО ДРУГИМ УЧРЕЖДЕНИЯМ, ЗАНЯТЫМ ДЕЛАМИ, ВРОДЕ БЫ ВОЗЛОЖЕННЫМИ, ПО НАШИМ ИСТОРИОГРАФИЧЕСКИМ ПРЕДСТАВЛЕНИЯМ, НА ПОЛКОВЫЕ ДВОРЫ. В ЭТИХ ФОРМУЛИРОВКАХ НЕСОМНЕННЫЙ ПРИОРИТЕТ ПРИНАДЛЕЖИТ ПОНЯТИЮ « КАНЦЕЛЯРИЯ СБОРА ПОДУШНЫХ ДЕНЕГ» - УЧРЕЖДЕНИЮ, ВОВСЕ НЕ УПОМИНАВШЕМУСЯ В ЗАКОНОДАТЕЛЬСТВЕ О ВОЕННО-ФИСКАЛЬНЫХ АДМИНИСТРАЦИЯХ. ЭТИ КАНЦЕЛЯРИИ, ОКАЗЫВАЕТСЯ, СУЩЕСТВУЮТ ПРИ ПОЛКАХ. ОНИ ВСТУПАЮТ В ПЕРЕПИСКУ МЕЖДУ СОБОЙ (« ПРОМЕМОРИЯ СУЗДАЛЬСКОЙ ПРАВИНЦИИ ДЕРПТСКОГО ПОЛКУ ИЗ КАНЦЕЛЯРИИ ЗБОРОВ ПОДУШНЫХ ДЕНЕГ ТОЙ ЖЕ ПРАВИНЦИИ ОТ КАНЦЕЛЯРИИ ГРАНОДЕРСКОГО КРОПОТОВА ПОЛКУ ИЗ КАНЦЕЛЯРИИ ЗБОРОВ ПОДУШНЫХ ДЕНЕГ» ОТ $1725 \Gamma^{32}$; «ПРОМЕМОРИЯ ТОБОЛЬСКОГО ГАРНИЗОНА САНКТПИТЕРБУРСКОГО ПЕХОТНОГО ПОЛКА ИЗ КАНЦЕЛЯРИИ ЗБОРА ПОДУШНЫХ ДЕНЕГ В ТЮМЕНСКУЮ КАНЦЕЛЯРИЮ ЗБОРА ПОДУШНЫХ ДЕНЕГ» ОТ 1725 И 1726 ГГ. ${ }^{33}$; «ПРОМЕМОРИЯ ИС КАНЦЕЛЯРИИ ПОДУШНОГО ЗБОРУ САНКТПИТЕРБУРГСКОГО АРМЕЙСКОГО ПЕХОТНОГО ПОЛКУ В КАНЦЕЛЯРИЮ ПОДУШНОГО ЗБОРУ ТОБОЛСКОГО ГВАРНИЗОНА САНКТПИТЕРБУРГСКОГО ПОЛКУ» ОТ 1726 Г. $^{34}$ ). К НИМ ОБРАЩАЮТСЯ УЧРЕЖДЕНИЯ ОБЩЕГО И СПЕЦИАЛЬНОГО УПРАВЛЕНИЯ (« ПРОМЕМОРИЯ ИЗ ТЮМЕНСКОЙ КАНЦЕЛЯРИИ СУДНЫХ ДЕЛ В КАНЦЕЛЯРИЮ СИБИРСКОГО ГАРНИЗОНА САНКТПИТЕРБУРГСКОГО ПОЛКУ ЗБОРА ПОДУШНЫХ ДЕНЕГ» ОТ $1726 \Gamma^{35}$; « ПРОМЕМОРИЯ ИС ТЮМЕНСКОЙ КАНЦЕЛЯРИИ ВОЕВОЦКОГО ПРАВЛЕНИЯ К ЗБОРУ ПОДУШНЫХ ДЕНЕГ СИБИРСКОГО ГВАРНИЗОНУ НА ТОБОЛСКОЙ ПОЛК» ОТ $\left.1731 \Gamma^{36}\right)$. ОНИ ВЫДАЮТ ПОДОРОЖНЫЕ (ИЗ ТЮМЕНСКОЙ КАНЦЕЛЯРИИ СБОРА ПОДУШНЫХ ДЕНЕГ КРЕСТЬЯНАМ ГРИГОРИЮ ЛУГАНИНОВУ И ИЛЬЕ КУЛАКОВУ, ПОСЛАННЫМ ЕЮ НА РОЗЫСК БЕГЛЫХ КРЕСТЬЯН, $1726 \Gamma^{37}$ ). ИХ ОБРАЩЕНИЯ ЗАСЛУШИВАЮТСЯ НА ЗАСЕДАНИЯХ ( ПРОТОКОЛ ЗАСЕДАНИЯ СИБИРСКОГО ОБЕР-БЕРГАМТА № 429. ТОГО Ж ЧИСЛА СЛУШАВ ПРОМЕМОРИЮ НОВОГОРОЦКОГО ПЕХОТНОГО ПОЛКУ ИС КАНЦЕЛЯРИИ ЗБОРА ПОДУШНЫХ ДЕНЕГ», 1726 Г. ${ }^{38}$ ; « ПРОТОКОЛ ЗАСЕДАНИЯ СИБИРСКОГО ОБЕР-БЕРГАМТА № 453. ТОГО Ж ЧИСЛА СЛУШАВ ПРОМЕМОРИЮ ИЗ ИСЕЦКА ИС КАНЦЕЛЯРИИ ПОДУШНОГО СБОРУ САНКТПИТЕРБУРХСКОГО АРМЕЙСКОГО ПЕХОТНОГО ПОЛКУ», 1726 Г. $\left.{ }^{39}\right)$.

НА ФОНЕ ЭТОГО МАССИВА ДОКУМЕНТОВ, В КОТОРЫХ КАНЦЕЛЯРИЯ СБОРА ПОДУШНЫХ ДЕНЕГ (КАНЦЕЛЯРИЯ ПОДУШНОГО СБОРА) ВЫСТУПАЕТ КАК ГЛАВНЫЙ АКТОР, ДАЖЕ ФОРМУЛИРОВКА «ИЗ ИСЕЦКА С ПОЛКОВОГО ДВОРА С ВЕЧНОЙ КВАРТИРЫ САНКТПИТЕРБУСКОГО АРМЕЙСКОГО ПЕХОТНОГО ПОЛКУ В ТЮМЕНЬ НА ПОЛКОВОЙ ДВОР ТОБОЛСКОГО ГВАРНИЗОНА САНКТПИТЕРБУРХСКОГО ПОЛКУ» (1726 Г.) ${ }^{40}$ НЕ ПОЗВОЛЯЕТ ОДНОЗНАЧНО РЕШИТЬ ВОПРОС ОБ ИНСТИТУЦИОНАЛЬНОМ СТАТУСЕ ПОЛКОВЫХ ДВОРОВ. ВОЗНИКАЕТ АЛЬТЕРНАТИВА: ЛИБО В СИСТЕМЕ МЕСТНЫХ ВОЕННО-ПОДАТНЫХ УЧРЕЖДЕНИЙ, СОЗДАННЫХ В ХОДЕ ПОДУШНОЙ РЕФОРМЫ, СУЩЕСТВОВАЛИ ДВЕ ИНСТАНЦИИ НЕЯСНОЙ СОПОДЧИНЕННОСТИ - ПОЛКОВОЙ ДВОР И КАНЦЕЛЯРИЯ СБОРА ПОДУШНЫХ ДЕНЕГ, ЛИБО ПОД ПОЛКОВЫМ ДВОРОМ СЛЕДУЕТ ПОНИМАТЬ МЕСТО, В КОТОРОМ РАСПОЛАГАЛОСЬ УЧРЕЖДЕНИЕ - ВЫШЕУПОМЯНУТАЯ КАНЦЕЛЯРИЯ. ЕСЛИ ДОПУСТИТЬ ПЕРВОЕ ${ }^{41}$, ТО ОСТАЕТСЯ НЕПОНЯТНЫМ, ПОЧЕМУ ПОЛКОВОЙ ДВОР РЕДКО ФИГУРИРУЕТ В КАЧЕСТВЕ УСТОЙЧИВОГО СУБЪЕКТА ДОКУМЕНТООБОРОТА. ВТОРОЕ ПРЕДПОЛОЖЕНИЕ ПРЕДСТАВЛЯЕТСЯ БОЛЕЕ ПРАВДОПОДОБНЫМ. НА ПОЛКОВОМ ДВОРЕ - В СВОЕОБРАЗНОМ ВОЕННОМ ГОРОДКЕ - РАСПОЛАГАЛИСЬ НЕСКОЛЬКО УЧРЕЖДЕНИЙ : ШТАБ ПОЛКА (ПОЛКОВОЕ УПРАВЛЕНИЕ В СОСТАВЕ ПОЛКОВНИКА, ШТАБНЫХ ОФИЦЕРОВ РАЗНОГО РАНГА, ТЫЛОВЫХ И ЮСТИЦКИХ ЧИНОВ), ПОЛКОВАЯ КАНЦЕЛЯРИЯ (УЧРЕЖДЕНИЕ, ВЕДШЕЕ 
ДЕЛОПРОИЗВОДСТВО ПОЛКА) И КАНЦЕЛЯРИЯ СБОРА ПОДУШНЫХ ДЕНЕГ (СПЕЦИАЛИЗИРОВАННОЕ МЕСТНОЕ УЧРЕЖДЕНИЕ ВОЕННО-ФИСКАЛЬНОГО УПРАВЛЕНИЯ). СТАРШИНСТВО СРЕДИ ЭТИХ ИНСТАНЦИЙ ПРИНАДЛЕЖАЛО, НЕСОМНЕННО, ШТАБУ, И В ПЕРВУЮ ОЧЕРЕДЬ, ПОЛКОВНИКУ. КАК НЕДВУСМЫСЛЕННО ПОКАЗЫВАЮТ СООТВЕТСТВУЮЩИЕ НОРМАТИВНО-ЗАКОНОДАТЕЛЬНЫЕ АКТЫ, ПОЛКОВНИК РАСПОЛАГАЛ ВСЕЙ ПОЛНОТОЙ ВЛАСТИ КАК ВНУТРИ ПОЛКА, ТАК И ПО ШИРОКОМУ КРУГУ ВОПРОСОВ, КОТОРЫЕ ПОРОДИЛА САМА СИТУАЦИЯ ПОСТОЯ ВОЙСК НА ЗИМНИХ КВАРТИРАХ И ВЗИМАНИЯ ПОДУШНОЙ ПОДАТИ. БЕЗУСЛОВНО ПОДЧИНЕННАЯ ПОЛКОВНИКУ ПОЛКОВАЯ КАНЦЕЛЯРИЯ (САМАЯ ПРОСТАЯ И БЛИЖАЙШАЯ АНАЛОГИЯ - ПОДЧИНЕННОСТЬ ВОЕВОДСКОЙ КАНЦЕЛЯРИИ ВОЕВОДЕ) МОГЛА, В СЛУЧАЕ НЕОБХОДИМОСТИ, ВТОРГАТЬСЯ В РЕШЕНИЕ ПРОБЛЕМ, ВЫХОДЯЩИХ ИЗ КРУГА ВНУТРЕННИХ ПОЛКОВЫХ ДЕЛ И ЗАНИМАТЬСЯ, НАПРИМЕР, ВОПРОСАМИ, СВЯЗАННЫМИ СО СБОРОМ ПОДУШНОГО НАЛОГА (КАК ПОКАЗЫВАЕТ, К ПРИМЕРУ, ПРОТОКОЛЬНАЯ ЗАПИСЬ СИБИРСКОГО ОБЕР-БЕРГАМТА, В КОТОРОМ СЛУШАЛАСЬ ПРОМЕМОРИЯ «ИС КАНЦЕЛЯРИИ НОВОГОРОЦКОГО ПОЛКУ» О РАССЫЛКЕ С ОФИЦЕРОМ ЭТОГО ПОЛКА « ДОНОШЕНИЕВ И КОПИЕВ С УКАЗОВ, ПОСЛАННЫХ... В ПРИПИСНЫЕ СЛОБОДЫ О ЗБОРЕ ПОДУШНЫХ ДЕНЕГ», $1726 \Gamma^{42}$ ).

КАНЦЕЛЯРИЯ ЖЕ СБОРА ПОДУШНЫХ ДЕНЕГ, ПО ПЕРВОНАЧАЛЬНОМУ ЗАМЫСЛУ, ДОЛЖНА БЫЛА СПЕЦИАЛИЗИРОВАТЬСЯ НА ОПЕРАЦИЯХ, СВЯЗАННЫХ С ОБСЛУЖИВАНИЕМ НОВОГО ПРЯМОГО НАЛОГА. ЭТО ЕЕ СОСТАВ ОПРЕДЕЛЯЛСЯ В ОБЩЕЙ ЧАСТИ П. 12 « ИНСТРУКЦИИ ПОЛКОВНИКУ» («КОГДА ПОЛК ОТ КВАРТИР БУДЕТ ВО ОТЛУЧЕНИИ, КОГО ОСТАВИТЬ В КВАРТИРАХ И КАК ПОСТУПАТЬ») : «...ОСТАВИТЬ В ТЕХ КВАРТИРАХ ДЛЯ ПРИЕМУ И ОТПРАВЛЕНИЯ ТЕХ ДЕНЕГ И ПРОЧЕГО, В ЕГО [ПОЛКОВНИКА. - Д.Р.] НЕБЫТНОСТЬ ОДНОГО КАПИТАНА И С НИМ ОДНОГО ПРАПОРЩИКА, ОДНОГО ПИСАРЯ И ОДНОГО КАПРАЛА ДА ДЛЯ КАРАУЛА И ПОСЫЛОК И ДЛЯ СЧЕТА ДЕНЕГ 16 ЧЕЛОВЕК РЯДОВЫХ...» ${ }^{43}$. В « ИНСТРУКЦИИ» У ЭТОЙ КОМАНДЫ НЕТ СОБСТВЕННОГО НАИМЕНОВАНИЯ ; ВЕРОЯТНО, ОНО ПОЯВИЛОСЬ УЖЕ В ХОДЕ ФУНКЦИОНИРОВАНИЯ ВОЕННЫХ АДМИНИСТРАЦИЙ И, КАК ЧАСТО БЫВАЛО В РУССКОЙ ПРАКТИКЕ ГОСУПРАВЛЕНИЯ, НЕ ОТЛИЧАЛОСЬ СТАБИЛЬНОСТЬЮ, ОБОЗНАЧАЯСЬ ТО КАК «КАНЦЕЛЯРИЯ СБОРА ПОДУШНЫХ ДЕНЕГ», ТО (РЕЖЕ) КАК « КАНЦЕЛЯРИЯ ПОДУШНОГО СБОРА» ИЛИ КАНЦЕЛЯРИЯ СМОТРЕНИЯ НАД ПОДУШНЫМ СБОРОМ». ТЕМ НЕ МЕНЕЕ, СУТЬ ОТ ЭТОГО НЕ МЕНЯЛАСЬ : РАЗЛИЧНЫЕ ФОРМЫ НАИМЕНОВАНИЯ ЧЕТКО ОПРЕДЕЛЯЛИ ГЛАВНОЕ ФУНКЦИОНАЛЬНОЕ ПРЕДНАЗНАЧЕНИЕ ЭТОГО ОРГАНА.

РАСПОЛАГАЯСЬ НА ПОЛКОВОМ ДВОРЕ, КАНЦЕЛЯРИЯ СБОРА ПОДУШНЫХ ДЕНЕГ МОГЛА (ПРИ ОТМЕЧЕННОЙ НЕУСТОЙЧИВОСТИ НАИМЕНОВАНИЯ) ИНСТИТУЦИОННО ОТОЖДЕСТВЛЯТЬСЯ С ПОЛКОВЫМ ДВОРОМ, ЧТО ПОРОЖДАЛО ФОРМУЛЫ ВРОДЕ ПРИВЕДЕННЫХ ВЫШЕ : «НА ПОЛКОВОЙ ДВОР К ПОДУШНОМУ СБОРУ». УТОЧНЕНИЕ ОКАЗЫВАЛОСЬ НЕОБХОДИМЫМ, КОГДА НА ПОЛКОВОМ ДВОРЕ ПРИСУТСТВОВАЛО СРАЗУ НЕСКОЛЬКО УЧРЕЖДЕНИЙ ВОЕННОЙ АДМИНИСТРАЦИИ. В СИТУАЦИЯХ ЖЕ, КОГДА НА ПОЛКОВОМ ДВОРЕ ОСТАВАЛАСЬ ОДНА ТОЛЬКО КАНЦЕЛЯРИЯ ПОДУШНОГО СБОРА, А ПОЛКОВНИК СО ШТАБОМ И ПОЛКОВОЙ КАНЦЕЛЯРИЕЙ ВЫБЫВАЛИ, ВОЗНИКАЛИ ФОРМУЛИРОВКИ, ПОДБНЫЕ УЖЕ ЦИТИРОВАВШЕЙСЯ : « ИЗ ИСЕЦКА С ПОЛКОВОГО ДВОРА С ВЕЧНОЙ КВАРТИРЫ САНКТПИТЕРБУРХСКОГО АРМЕЙСКОГО ПЕХОТНОГО ПОЛКУ В ТЮМЕНЬ НА ПОЛКОВОЙ ДВОР ТОБОЛСКОГО ГВАРНИЗОНА САНКТПИТЕРБУРХСКОГО ПОЛКУ» (1726 Г.) 44. В ДАННОМ СЛУЧАЕ ПРОИСХОДИТ ПОЛНОЕ ОТОЖДЕСТВЛЕНИЕ КАНЦЕЛЯРИИ ПОДУШНОГО СБОРА И ПОЛКОВОГО ДВОРА (В ПОДАВЛЯЮЩЕМ БОЛЬШИНСТВЕ ПРИМЕРОВ ПЕРЕПИСКИ МЕЖДУ ЭТИМИ ПОЛКАМИ ФИГУРИРУЮТ КАНЦЕЛЯРИИ, А НЕ ДВОРЫ). 
ИСПОЛЬЗОВАНИЕ ТАКОЙ ФОРМУЛИРОВКИ, ЯВЛЯВШЕЙСЯ, СТРОГО ГОВОРЯ, ОТСТУПЛЕНИЕМ ОТ НОРМЫ, ВЫРАБОТАННОЙ КАНЦЕЛЯРСКОЙ ПРАКТИКОЙ, МОЖЕТ БЫТЬ ТАКЖЕ ОБЪЯСНЕНО СМЕНОЙ ПОДЬЯЧЕГО, ВЕДШЕГО ДЕЛОПРОИЗВОДСТВО, ЕГО СТИЛЕВЫМИ И ТЕРМИНОЛОГИЧЕСКИМИ ПРЕДПОЧТЕНИЯМИ.

ПРИВЕДЕННЫЕ СООБРАЖЕНИЯ, ОСНОВАННЫЕ НА ИЗУЧЕНИИ ДЕЛОПРОИЗВОДСТВЕННОЙ ДОКУМЕНТАЦИИ ВОЕННО-ПОДАТНЫХ АДМИНИСТРАЦИЙ, ГАРМОНИЧНО СОЧЕТАЮТСЯ С ДАННЫМИ НОРМАТИВНО-ЗАКОНОДАТЕЛЬНЫХ АКТОВ, РАЗОБРАННЫХ ВЫШЕ. ПРИ ВСЕХ ВОЗМОЖНЫХ ВАРИАЦИЯХ, КОТОРЫЕ ПОТЕНЦИАЛЬНО НЕСЕТ В СЕБЕ ЕЩЕ НЕ ВЫЯВЛЕННЫЙ КРУГ ДОКУМЕНТАЛЬНЫХ КОМПЛЕКСОВ АНАЛОГИЧНОГО ПРОИСХОЖДЕНИЯ, КАЖЕТСЯ НАИБОЛЕЕ ОБОСНОВАННЫМ ПРИЗНАТЬ, ЧТО ПОЛКОВОЙ ДВОР КАК « МЕСТНОЕ УЧРЕЖДЕНИЕ ВОЕННОГО УПРАВЛЕНИЯ» ЕСТЬ ИСТОРИОГРАФИЧЕСКИЙ МИФ. СТРУКТУРА МЕСТНЫХ УЧРЕЖДЕНИЙ ВОЕННОГО УПРАВЛЕНИЯ, ВОЗНИКШАЯ В ХОДЕ ВВЕДЕНИЯ ПОДУШНОЙ ПОДАТИ, БЫЛА СЛОЖНЕЕ. ОНА ВКЛЮЧАЛА ПОЛКОВНИКА С ЕГО ШТАБОМ, ПОЛКОВУЮ КАНЦЕЛЯРИЮ И КАНЦЕЛЯРИЮ СБОРА ПОДУШНЫХ ДЕНЕГ. ПОСЛЕДНЯЯ, РАСПОЛАГАВШАЯСЯ, ВМЕСТЕ С ВЫШЕУКАЗАННЫМИ, НА ПОЛКОВОМ ДВОРЕ, ИГРАЛА В ЭТОМ УПРАВЛЕНИИ КЛЮЧЕВУЮ РОЛЬ, КОТОРАЯ ПРИОБРЕТАЛА ЕДВА ЛИ НЕ ВСЕОБЪЕМЛЮЩИЙ ХАРАКТЕР В ТЕХ СЛУЧАЯХ, КОГДА ПО КАКИМ-ТО ПРИЧИНАМ ПОЛКОВНИК С ОФИЦЕРАМИ ПОКИДАЛИ ПОЛКОВОЙ ДВОР - ЦЕНТР СВОЕГО ПОЛКОВОГО ДИСТРИКТА.

РАЗВЕРТЫВАНИЕ В РЕГИОНАХ ШИРОКОЙ СЕТИ ВОЕННЫХ УЧРЕЖДЕНИЙ САМЫМ СЕРЬЕЗНЫМ ОБРАЗОМ ИЗМЕНИЛО АДМИНИСТРАТИВНЫЙ ЛАНДШАФТ РОССИИ. СТРОГО ГОВОРЯ, ЭТО СТАЛО ОЩУЩАТЬСЯ ЕЩЕ ДО ТОГО, КАК НА ТЕРРИТОРИЯХ ГУБЕРНИЙ И ПРОВИНЦИЙ ЗАСТУЧАЛИ ТОПОРЫ, ВОЗВЕЩАЯ НАЧАЛО СТРОИТЕЛЬСТВА СОЛДАТСКИХ СЛОБОД И ПОЛКОВЫХ ДВОРОВ, - С ТОГО МОМЕНТА, КОГДА ТЕКУЩАЯ ЖИЗНЬ ОБЛАСТИ ОКАЗАЛАСЬ ПОДЧИНЕНА ДИКТАТУ ПЕРЕПИСНЫХ КАНЦЕЛЯРИЙ - ОРГАНОВ ЧРЕЗВЫЧАЙНОГО, ИЛИ ЭКСТРАОРДИНАРНОГО УПРАВЛЕНИЯ. ИХ БЕСЦЕРЕМОННОЕ И ВЛАСТНОЕ ВТОРЖЕНИЕ В СФЕРУ КОМПЕТЕНЦИИ ОРДИНАРНЫХ ОРГАНОВ ОБЩЕГО И СПЕЦИАЛЬНОГО УПРАВЛЕНИЯ, СОЗДАННЫХ В ХОДЕ ВТОРОЙ ОБЛАСТНОЙ РЕФОРМЫ, ПРЕКРАСНО ОПИСАЛ М.М.БОГОСЛОВСКИЙ. ХАРАКТЕРИЗУЯ ПОЛОЖЕНИЕ ПРОВИНЦИАЛЬНОЙ АДМИНИСТРАЦИИ В 1720-ХГГ., ИСТОРИК ЗАМЕТИЛ: «...КРОМЕ ПРИСТАВЛЕННОГО К НЕЙ ГВАРДЕЙЦА, НАДЗОР КОТОРОГО ТЯГОТЕЛ НАД НЕЮ [РЕЧЬ ИДЕТ О Т.Н. ГВАРДЕЙЦАХ- «ПОНУДИТЕЛЯХ». - Д.Р.] СВЕРХУ ЕЕ ДАВИЛ ВЛАСТНЫЙ И ТРЕБОВАТЕЛЬНЫЙ ПЕРЕПИСЧИК, С БОКОВ СЖИМАЛИ ПОЛКОВЫЕ КОМАНДИРЫ» ${ }^{45}$.

ПРИ ВСЕМ ВСЕВЛАСТИИ ПЕРЕПИСНЫХ КАНЦЕЛЯРИЙ, ПАРАЛИЗОВАВШИХ, ПО СЛОВАМ БОГОСЛОВСКОГО, ПРОВИНЦИАЛЬНЫЕ АДМИНИСТРАЦИИ И КОНТРОЛИРОВАВШИХ ДЕЯТЕЛЬНОСТЬ ПОЛКОВЫХ ОРГАНОВ МЕСТНОГО УПРАВЛЕНИЯ, ОНИ МЫСЛИЛИСЬ ЗАКОНАДАТЕЛЕМ КАК ВРЕМЕННОЕ ЯВЛЕНИЕ УПРАВЛЕНЧЕСКОЙ СИСТЕМЫ. НА ЭТО УКАЗЫВАЕТ САМ ПРОЦЕСС ЗАКОНОДАТЕЛЬНОГО ОБЕСПЕЧЕНИЯ ДЕЯТЕЛЬНОСТИ ПЕРЕПИСНЫХ КАНЦЕЛЯРИЙ : ОНО ФОРМИРОВАЛОСЬ КАЗУИСТИЧЕСКИ, НА ПРОТЯЖЕНИИ ОТНОСИТЕЛЬНОГО ДЛИТЕЛЬНОГО (ПО МЕРКАМ ДИНАМИЧНОГО ПЕТРОВСКОГО ЦАРСТВОВАНИЯ) ВРЕМЕНИ. ПОСЛЕ ЗАВЕРШЕНИЯ ПЕРЕПИСИ, ПЕРЕПИСНЫЕ КАНЦЕЛЯРИИ ПРЕДПОЛАГАЛОСЬ ЛИКВИДИРОВАТЬ, ЧТО В КОНЦЕ КОНЦОВ И ПРОИЗОШЛО. ПОЛКОВЫЕ АДМИНИСТРАЦИИ, НАПРОТИВ, ВЫСТРАИВАЛИСЬ КАК ПОСТОЯННЫЕ УЧРЕЖДЕНИЯ. ПЕТР, СУДЯ ПО ВСЕМУ, ПРЕДПОЛАГАЛ, ЧТО ИХ ВЕК БУДЕТ ДОЛОГ И ИМЕННО ПОЭТОМУ СНАБДИЛ 
ЭТИ УЧРЕЖДЕНИЯ ЕДИНОВРЕМЕННО ОПУБЛИКОВАННЫМ МАССИВОМ НОРМАТИВОВ, НОСИВШИХ СТОЛЬ ЖЕ «ПАКЕТНЫЙ» ХАРАКТЕР, КАКОВОЙ БЫЛ ПРИСУЩ НОРМАТИВАМ ФУНКЦИОНИРОВАНИЯ ОБЛАСТНЫХ АДМИНИСТРАЦИЙ 1719 Г. РАБОТУ МЕСТНЫХ ОРГАНОВ ВОЕННОГО УПРАВЛЕНИЯ И ОРГАНОВ ПРОВИНЦИАЛЬНОГО УПРАВЛЕНИЯ, ДЕМОНСТРИРУЕТ РЕДУКЦИЮ ПОЛНОМОЧИЙ ВТОРЫХ В ПОЛЬЗУ ПЕРВЫХ, ПО ЦЕЛОМУ РЯДУ ОСНОВНЫХ НАПРАВЛЕНИЙ. В ЭТОМ ЛЕГКО УБЕДИТЬСЯ, РАССМОТРЕВ ПОЛНОМОЧИЯ ПОЛКОВНИКА И ЕГО ОФИЦЕРОВ, ИЗЛОЖЕННЫЕ В ИНСТРУКЦИЯХ И « ПЛАКАТЕ» 1724 Г., В СРАВНЕНИИ С ПОЛНОМОЧИЯМИ ВОЕВОД/ГУБЕРНАТОРОВ И ЧИНОВ ПРОВИНЦИАЛЬНОЙ АДМИНИСТРАЦИИ 1719 Г. ДАЖЕ С ФОРМАЛЬНО-ЮРИДИЧЕСКОЙ ТОЧКИ ЗРЕНИЯ ЭТО СРАВНЕНИЕ ОКАЗЫВАЕТСЯ НЕ В ПОЛЬЗУ ПОСЛЕДНИХ. ИНСТРУКЦИИ 1719 Г. В БОЛЬШИНСТВЕ СВОЕМ СОДЕРЖАТ ОБЯЗЫВАЮЩИЕ НОРМЫ ПРАВА. ПО БУКВЕ И ДУХУ ОНИ В ПРЕДЕЛЬНОЙ СТЕПЕНИ СТАВЯТ ЧИНЫ МЕСТНЫХ АДМИНИСТРАЦИЙ В ПОЛОЖЕНИЕ МЕХАНИЧЕСКИХ ИСПОЛНИТЕЛЕЙ ВОЛИ ЦЕНТРАЛЬНОЙ ВЛАСТИ, ПОСТОЯННО НАПОМИНАЯ ИМ : «ТЫ ДОЛЖЕН». НАПРОТИВ, ИНСТРУКЦИИ 1724 Г., ОБРАЩЕННЫЕ К ПОЛКОВНИКУ И ОФИЦЕРАМ ПОЛКА, НАПОЛНЕНЫ УПОЛНОМОЧИВАЮЩИМИ НОРМАМИ ПРАВА, ПРЕДОСТАВЛЯЮЩИМИ ВОЗМОЖНОСТЬ СОВЕРШАТЬ ТЕ ИЛИ ИНЫЕ ДЕЙСТВИЯ.

КАК УПОМИНАЛОСЬ ВЫШЕ, ОРГАНИЗУЯ РАССЕЛЕНИЕ ВОЙСК И ВВЕДЕНИЕ ПОДУШНОГО НАЛОГА, ПЕТР НЕ НАМЕРЕВАЛСЯ ВОВСЕ ИСКЛЮЧИТЬ ИЗ ЭТИХ ПРОЦЕССОВ РУКОВОДСТВО ГУБЕРНИЙ И ПРОВИНЦИЙ. ВОЕВОДЫ, В СООТВЕТСТВИИ С ИНСТРУКЦИЕЙ 1719 Г., НАДЕЛЯЛИСЬ ДОСТАТОЧНО ШИРОКИМИ ВОЗМОЖНОСТЯМИ ДЛЯ УЧАСТИЯ В ПРОВОДИМОЙ РЕФОРМЕ И ДАЖЕ ИМЕЛИ ОПРЕДЕЛЕННЫЕ КОНТРОЛИРУЮЩИЕ ФУНКЦИИ. ВОЕВОДЕ ОТВОДИЛАСЬ РОЛЬ ТРЕТЕЙСКОГО СУДЬИ « В ОБИДАХ, ССОРАХ И НЕПОРЯДКАХ», КОТОРЫЕ МОГЛИ ВОЗНИКАТЬ МЕЖДУ ВОЕННЫМИ И ТЯГЛЕЦАМИ; ЕМУ ВМЕНЯЛСЯ НАДЗОР ЗА ДЕЯТЕЛЬНОСТЬЮ КОМИССАРОВ ОТ ЗЕМЛИ В ПРОЦЕССЕ РАСКЛАДКИ И СБОРА ПОДУШНОЙ ПОДАТИ ; ОН БЫЛ УПОЛНОМОЧЕН СЛЕДИТЬ, ЧТОБЫ КРЕСТЬЯНЕ « НЕ БЫЛИ ОБИДИМЫ» ОТ ВОЕННЫХ, КАК ПРИ СБОРЕ СРЕДСТВ НА ИХ «ЖАЛОВАНЬЕ И ПРОТЧЕЕ», ТАК И ПРИ ОСУЩЕСТВЛЕНИИ РЕКРУТСКИХ НАБОРОВ ${ }^{46}$. ОДНАКО ЭТИ АДМИНИСТРАТИВНЫЕ ПРАВА БЫЛИ СВЕДЕНЫ НА НЕТ ИНСТРУКЦИЯМИ 1724 Г., ИЗЪЯВШИМИ ВЕСЬ КРУГ ВОПРОСОВ, СВЯЗАННЫХ СО СБОРОМ ПОДУШНОГО НАЛОГА И ОТПРАВЛЕНИЕМ РЕКРУТСКОЙ ПОВИННОСТИ, В ПОЛЬЗУ ВОЕННЫХ УЧРЕЖДЕНИЙ. ДАЖЕ КОНТРОЛЬ НАД КОМИССАРАМИ ОТ ЗЕМЛИ - ПРЕДСТАВИТЕЛЯМИ МЕСТНОГО НАСЕЛЕНИЯ, ПОДВЕДОМСТВЕННОГО ОБЩИМ АДМИНИСТРАЦИЯМ - ОКАЗАЛСЯ В РУКАХ ПОЛКОВНИКОВ. НОРМАТИВНО, ИМЕННО НА НИХ БЫЛИ ВОЗЛОЖЕНЫ ЗАБОТЫ О ПРОВЕДЕНИИ УЕЗДНЫХ СЪЕЗДОВ ПО ВЫБОРУ КОМИССАРОВ, А ВОПРОС НАКАЗАНИЯ НЕРАДИВЫХ КОМИССАРОВ ТОЛКОВАЛСЯ ТАК РАСПЛЫВЧАТО, ЧТО ПРИХОДИЛОСЬ РЕШАТЬ ЕГО ПО СИТУАЦИИ ${ }^{47}$. ВОЕВОДУ ПОЛНОСТЬЮ УСТРАНЯЛИ ОТ КОНТРОЛЯ ЗА СНАБЖЕНИЕМ ВОЙСК, ПРОХОДЯЩИХ МАРШЕМ ЧЕРЕЗ ВВЕРЕННУЮ ЕМУ ПРОВИНЦИЮ ${ }^{48}$.

ВОПИЮЩИМ ИЗЪЯТИЯМ ПОДВЕРГЛИСЬ ПРЕРОГАТИВЫ ОБЛАСТНЫХ АДМИНИСТРАЦИЙ В СФЕРЕ ОБЩЕГО АДМИНИСТРИРОВАНИЯ, ПОЛИЦЕЙСКОГО КОНТРОЛЯ И СУДЕБНЫХ ПОЛНОМОЧИЙ. « ИНСТРУКЦИЯ» 1719 Г. ВМЕНЯЛА В ОБЯЗАННОСТИ ВОЕВОД ПОПЕЧЕНИЕ О ЗЕМСКОЙ ПОЛИЦИИ ; ОБЩИЙ НАДЗОР ЗА СОБЛЮДЕНИЕМ ЗАКОННОСТИ (СОДЕРЖАТЬ ВСЕХ ПОДДАННЫХ СВОЕЙ ПРОВИНЦИИ « ПО ГОСУДАРЕВЫМ УЛОЖЕНЬЯМ» ; « СТАРАТИСЯ, ЧТОБ НИКОМУ НАСИЛИЯ И ГРАБЕЖА ЧИНЕНО НЕ БЫЛО») ; БОРЬБУ С « ВОРОВСТВОМ, И ВСЯКИМИ РАЗБОЯМИ И ПРЕСТУПЛЕНИЯМИ» ; ДАВАЛА ПРАВО ПРОВОДИТЬ РОЗЫСК И ОСУЩЕСТВЛЯТЬ 
НАКАЗАНИЯ ${ }^{49}$. СОВЕРШЕННО ИНАЧЕ РАССТАВЛЯЛИ АКЦЕНТЫ НОРМАТИВНО-ЗАКОНОДАТЕЛЬНЫЕ АКТЫ 1724 Г. БОРЬБА С ПРЕСТУПНОСТЬЮ НА ТЕРРИТОРИИ ПОЛКОВОГО ДИСТРИКТА ОТДАВАЛСЬ В ИСКЛЮЧИТЕЛЬНУЮ ЮРИСДИКЦИЮ ПОЛКОВНИКОВ (ШИРЕ - ВОЕННОЙ АДМИНИСТРАЦИИ). ЭТА НОРМА, ПРОВОЗГЛАШЕННАЯ В ОБЩЕМ В П. 11 Ч. 2 « ИНСТРУКЦИИ ПОЛКОВНИКУ», СУЩЕСТВЕННО УТОЧНЯЛАСЬ В УКАЗЕ «О ДОЛЖНОСТИ ПОЛКОВНИКА», ПЕРЕЧЕРКИВАЯ ВСЯКОЕ ПРАВО ВОЕВОД В ПОДОБНЫХ ДЕЛАХ. ПОЛКОВНИК ПРЯМО ОБЯЗЫВАЛСЯ ОХРАНЯТЬ «ОТ ВСЯКИХ ОБИД И НАЛОГ» « УЕЗДНЫХ ЛЮДЕЙ», А ИХ ПРИТЕСНИТЕЛЕЙ» « АРЕСТОВЫВАТЬ И ПИСАТЬ» (П. 2) 50 . ПОД КОНТРОЛЬ ВОЕННОЙ АДМИНИСТРАЦИИ ПОПАДАЛИ ТАКЖЕ ТАКИЕ ВАЖНЕЙШИЕ АДМИНИСТРАТИВНО-ПОЛИЦЕЙСКИЕ ФУНКЦИИ, КАК РОЗЫСК И ПОИМКА БЕГЛЫХ, ПРЕСЕЧЕНИЕ УКРЫВАТЕЛЬСТВА БЕГЛЕЦОВ (ВКЛЮЧАЯ НАЛОЖЕНИЕ И ВЗЫСКАНИЕ ШТРАФОВ С УКРЫВАТЕЛЕЙ), ПРОЦЕДУРА ВЫДАЧИ ПАСПОРТОВ И ДАЖЕ НАДЗОР ЗА ПЕРЕВОДОМ КРЕСТЬЯН ВНУТРИ ВЛАДЕНИЙ ОДНОГО ДУШЕВЛАДЕЛЬЦА. ВОЕВОДАМ И ГУБЕРНАТОРАМ ВО ВСЕХ ЭТИХ ДЕЙСТВИЯХ ОТВОДИЛАСЬ ВСПОМОГАТЕЛЬНАЯ РОЛЬ. НАПРИМЕР, ПОЙМАННЫХ БЕГЛЫХ ПРЕДПИСЫВАЛОСЬ СДАВАТЬ ПОД ВРЕМЕННОЕ СОДЕРЖАНИЕ ВОЕВОДАМ; НАДО ПОЛАГАТЬ, ТАКАЯ УСТУПКА БЫЛА СДЕЛАНА ЛИШЬ ОТТОГО, ЧТО В РАСПОРЯЖЕНИИ ПОСЛЕДНИХ ИМЕЛИСЬ ПОДХОДЯЩИЕ ДЛЯ ЭТОГО ТЮРЕМНЫЕ ПОМЕЩЕНИЯ ${ }^{51}$. СУДЕБНАЯ ЮРИСДИКЦИЯ ВОЕВОД СВОДИЛАСЬ К ПРАВУ СОУЧАСТИЯ В СОВМЕСТНОМ РАЗБИРАТЕЛЬСТВЕ КОНФЛИКТОВ И ПРАВОНАРУШЕНИЙ, ВОЗНИКАВШИХ ПРИ СТОЛКНОВЕНИЯХ СУБЪЕКТОВ РАЗНОЙ ВЕДОМСТВЕННОЙ ПРИНАДЛЕЖНОСТИ (МЕЖДУ ВОЕННЫМИ И ГРАЖДАНСКИМИ ЛИЦАМИ $)^{52}$ - ПОРЯДОК, СПРАВЕДЛИВО НАПОМНИВШИЙ М.М. БОГОСЛОВСКОМУ НОРМЫ «СМЕСТНОГО» ИЛИ « ВОПЧЕГО» СУДА, ПРАКТИКОВАВШЕГОСЯ В СРЕДНЕВЕКОВОЙ РОССИИ ${ }^{53}$.

41 НАКОНЕЦ, ЗАКОНОДАТЕЛЬСТВО 1724 Г. ПОЗВОЛИЛО ВОЕННЫМ УЧРЕЖДЕНИЯМ ВТОРГНУТЬСЯ НЕПОСРЕДСТВЕННО ВО ВНУТРИВЕДОМСТВЕННЫЕ РАСПОРЯЖЕНИЯ ОБЩИХ АДМИНИСТРАЦИЙ : ПОД КОНТРОЛЬ ПОЛКОВНИКОВ БЫЛА ПОСТАВЛЕНА ПОСЫЛКА ВОЕВОДОЙ ПРИКАЗНЫХ ЛЮДЕЙ С ПОРУЧЕНИЯМИ ПО ТЕРРИТОРИИ УЕЗДА. НАДО ЗАМЕТИТЬ, ЧТО ПОЕЗКИ ПОДЬЯЧИХ ПО СЕЛАМ И ДЕРЕВНЯМ ЗАЧАСТУЮ СОПРОВОЖДАЛИСЬ ВЫМОГАТЕЛЬСТВОМ С НАСЕЛЕНИЯ «КОРМОВ». ТАКИЕ ЭКСПЕДИЦИИ ВСЕГДА ХАРАКТЕРИЗОВАЛИСЬ КАК БЕЗДЕЛЬНЫЕ» И ВРЕМЯ ОТ ВРЕМЕНИ ПОПАДАЛИ В ПОЛЕ ЗРЕНИЯ МЕСТНЫХ ВЛАСТЕЙ, ПЫТАВШИХСЯ ЕСЛИ НЕ ЗАПРЕТИТЬ, ТО ОГРАНИЧИТЬ ИХ. ОДНАКО «ИНСТРУКЦИЯ ПОЛКОВНИКАМ» ПРЕДПИСАЛА ОБЯЗАТЕЛЬНОЕ СОГЛАСОВАНИЕ ВСЕХ КОМАНДИРОВОК ПРИКАЗНЫХ С ПОЛКОВЫМ КОМАНДИРОМ РАСКВАРТИРОВАННОЙ В МЕСТНОСТИ ЧАСТИ; ПОСЛЕДНЕМУ СЛЕДОВАЛО ВЫДЕЛЯТЬ В СОПРОВОЖДЕНИЕ ПОДЬЯЧЕМУ СВОИХ СОЛДАТ, ЧТОБЫ ЧИНОВНИКИ «ПОРЯДОЧНО И БЕЗ РАЗЗОРЕНИЯ ЧИНИЛИ, А НЕ ТАК КАК ПРЕЖДЕ БЫЛО РАЗЗОРЕНИЕ ОТ ПОСЛАННЫХ ${ }^{54}$. ОСТАВИМ В СТОРОНЕ РАССУЖДЕНИЯ О ТОМ, НАСКОЛЬКО ДЕЙСТВЕН БЫЛ НОВЫЙ ПОРЯДОК ; ДОКУМЕНТЫ СВИДЕТЕЛЬСТВУЮТ, ЧТО ТАКИЕ КОМАНДИРОВКИ, «УСИЛЕННЫЕ» СОЛДАТАМИ-НАДЗИРАТЕЛЯМИ, ПРИВОДИЛИ К ЕЩЕ БОЛЬШИМ «РАЗЗОРЕНИЯМ» КРЕСТЬЯН. В ЭТОЙ НОРМЕ ВАЖНО ТО, ЧТО ОНА, КАК И ВСЕ ОСТАЛЬНЫЕ, ОКАЗАЛАСЬ НАПРАВЛЕННОЙ НА СУЖЕНИЕ ПОЛНОМОЧИЙ МЕСТНЫХ АДМИНИСТРАЦИЙ. В КОНЕЧНОМ ИТОГЕ, ДАЖЕ ПЕРВЫЕ ЛИЦА ГУБЕРНИЙ И ПРОВИНЦИЙ ОКАЗАЛИСЬ ПОДНАДЗОРНЫ ПОЛКОВЫМ КОМАНДИРАМ. УКАЗ О ДОЛЖНОСТИ ПОЛКОВНИКА» УСТАНАВЛИВАЛ ПРАВО КОМАНДИРА РАСКВАРТИРОВАННОГО ПОЛКА ОТСЛЕЖИВАТЬ, НАСКОЛЬКО УСЕРДНО ГУБЕРНАТОРЫ И ВОЕВОДЫ ИСПОЛНЯЮТ ПОСЫЛАЕМЫЕ К НИМ СЕНАТСКИЕ И КОЛЛЕЖСКИЕ УКАЗЫ; ОБЯЗЫВАЛ ЕГО ДОКЛАДЫВАТЬ В ВЫШЕСТОЯЩИЕ ИНСТАНЦИИ О НЕРАДИВЫХ 
ГРАЖДАНСКИХ НАЧАЛЬНИКАХ И ДАЖЕ ДАВАЛ ПОЛНОМОЧИЕ «ЧИНИТЬ НА НИХ ВЗЫСКАНИЕ», ЕСЛИ НА ТО БУДЕТ ПОЛУЧЕНА САНКЦИЯ СООТВЕТСТВУЮЩЕГО ЦЕНТРАЛЬНОГО ОРГАНА ${ }^{55}$.

ТАКИМ ОБРАЗОМ, УЖЕ ИЗНАЧАЛЬНО, НА ЗАКОНОДАТЕЛЬНО-НОРМАТИВНОМ УРОВНЕ ВОЕННЫМ АДМИНИСТРАЦИЯМ БЫЛИ ПРИДАНЫ ТАКИЕ ШИРОКИЕ ПОЛНОМОЧИЯ, ЧТО ОНИ ВПОЛНЕ МОГЛИ ЧУВСТВОВАТЬ СЕБЯ ХОЗЯЕВАМИ ПОЛОЖЕНИЯ. ДРАМАТИЗМ СИТУАЦИИ ЗАКЛЮЧАЛСЯ ЕЩЕ И В ТОМ, ЧТО УСТАНОВЛЕНИЯ 1724 Г. НЕ ОТМЕНЯЛИ ИНСТРУКЦИИ 1719 Г. ТАКАЯ ЗАКОНОДАТЕЛЬНАЯ НЕСОГЛАСОВАННОСТЬ, ОЧЕНЬ ХАРАКТЕРНАЯ ДЛЯ ПЕТРОВСКОГО ЦАРСТВОВАНИЯ, СОЗДАВАЛА МНОГОЧИСЛЕННЫЕ ЗОНЫ НАПРЯЖЕННОСТИ КАК В МЕЖВЕДОМСТВЕННЫХ КОНТАКТАХ, ТАК И В ОТНОШЕНИЯХ МЕЖДУ РАЗЛИЧНЫМИ ВЛАСТНЫМИ ИНСТАНЦИЯМИ И НАСЕЛЕНИЕМ. РАЗУМЕЕТСЯ, ОНА ДАВАЛА ПРОСТОР И ДЛЯ ЗЛОУПОТРЕБЛЕНИЙ, ОСТАВЛЯЯ РАЗРЕШЕНИЕ КОНФЛИКТНЫХ СИТУАЦИЙ НА ВОЛЮ СЛУЧАЯ, УСИЛИВАЯ ТРАДИЦИОННОЕ « КТО СИЛЬНЕЕ, ТОТ И ПРАВ». В ЭТОМ ОТНОШЕНИИ, ЧАЩЕ ВСЕГО БРАЛИ ВЕРХ ОРГАНЫ ВОЕННОГО УПРАВЛЕНИЯ. НА ИХ СТОРОНЕ БЫЛИ НЕ ТОЛЬКО ПОКРОВИТЕЛЬСТВО МОГУЩЕСТВЕННЫХ ПЕРЕПИСЧИКОВ И ВОЕННОЙ КОЛЛЕГИИ, НО И ЛЮДСКИЕ РЕСУРСЫ - ВОИНСКИЕ КОНТИНГЕНТЫ. ПРАКТИКА ПОКАЗЫВАЕТ, ЧТО В ПОВСЕДНЕВНОЙ ЖИЗНИ ВЛАСТЬ НА МЕСТАХ ОБЫЧНО РЕАЛИЗОВЫВАЛАСЬ ДАЖЕ НЕ ПОЛКОВНИКАМИ, А ОФИЦЕРАМИ, ОТВЕТСТВЕННЫМИ ЗА СБОР ПОДУШНОЙ ПОДАТИ ГЛАВАМИ КАНЦЕЛЯРИЙ ПОДУШНЫХ СБОРОВ, КВАРТИРОВАВШИХ НА ПОЛКОВЫХ ДВОРАХ. ИМЕННО ЭТИ МАЙОРЫ, КАПИТАНЫ И ПРАПОРЩИКИ СТАЛИ НАСТОЯЩИМИ КОНКУРЕНТАМИ ВОЕВОД В ЕЖЕДНЕВНОМ БЫТУ, ПРЕДЕЛЬНО ШИРОКО ТРАКТУЯ В СВОЮ ПОЛЬЗУ ПОЛНОМОЧИЯ, ПРЕДОСТАВЛЕННЫЕ ЗАКОНОДАТЕЛЕМ.

ПОЯВЛЕНИЕ В АДМИНИСТРАТИВНОЙ ЖИЗНИ ПЕТРОВСКОЙ РОССИИ ВОЕННО-ПОДАТНЫХ УЧРЕЖДЕНИЙ, de facto ПРЕВРАТИВШИХСЯ В НАИБОЛЕЕ ВЛИЯТЕЛЬНЫЕ ОРГАНЫ МЕСТНОЙ ВЛАСТИ, ПОЗВОЛЯЕТ ПОСТАВИТЬ ВОПРОС О ХАРАКТЕРЕ СИСТЕМЫ УПРАВЛЕНИЯ СТРАНОЙ В ЦЕЛОМ. ГОВОРЯ О ТОРЖЕСТВЕ КАМЕРАЛИСТСКИХ ИДЕЙ, О ПОСТРОЕНИИ КОЛЛЕЖСКОЙ МОДЕЛИ УПРАВЛЕНИЯ В ПОСЛЕДНИЕ ГОДЫ ЦАРСТВОВАНИЯ ПЕТРА І, МЫ, ВИДИМО, ДОЛЖНЫ УЧИТЫВАТЬ, ЧТО, КАК И НА БОЛЕЕ РАННИХ ЭТАПАХ, ВСЕ ЭТО ОСТАВАЛОСЬ, СКОРЕЕ, УПОВАНИЕМ, НЕЖЕЛИ РЕАЛЬНОСТЬЮ, НЕИЗМЕННО УСТУПАЯ (ПОД ВЛИЯНИЕМ ТРАДИЦИИ И СИЮМИНУТНЫХ ЗАДАЧ) БОЛЕЕ ПРОСТЫМ УПРАВЛЕНЧЕСКИМ ПРАКТИКАМ. ЭТА МЫСЛЬ ПОДТВЕРЖДАЕТСЯ ТАКЖЕ ТЕМ, ЧТО ПРЕЕМНИКИ ПЕТРА ОЧЕНЬ ЛЕГКО И БЫСТРО ЛИКВИДИРОВАЛИ СОЗДАННЫЕ ИМ РАЗНООБРАЗНЫЕ ОРГАНЫ МЕСТНОЙ ВЛАСТИ, ОСНОВАННЫЕ НА ПРИНЦИПАХ КАМЕРАЛИСТСКОГО УПРАВЛЕНИЯ, НО ОТНОСИТЕЛЬНО ДОЛГО НЕ МОГЛИ ОТКАЗАТЬСЯ ОТ НЕХИТРОЙ И, ВЕРОЯТНО, БОЛЕЕ ЭФФЕКТИВНОЙ СИСТЕМЫ ВОЕННО-ПОДАТНЫХ УЧРЕЖДЕНИЙ. ТОЛЬКО К 1736 Г., УЖЕ В ЦАРСТВОВАНИЕ АННЫ, ПРАВИТЕЛЬСТВО СУМЕЛО ПРИСПОСОБИТЬ ВОЕННЫЕ ИНСТАНЦИИ К ОБЩЕЙ СИСТЕМЕ ПРЕДЕЛЬНО УПРОЩЕННОЙ ГРАЖДАНСКОЙ АДМИНИСТРАЦИИ, ПОДЧИНИВ ОФИЦЕРОВ ПРИ СБОРЕ ПОДУШНОЙ ПОДАТИ (ЛИШЕННЫХ СИЛЬНОГО ИСПОЛНИТЕЛЬНОГО АППАРАТА) УЕЗДНЫМ ВОЕВОДАМ. 


\section{NOTES}

1. НА ЭТО ЕСТЬ ПРЯМЫЕ УКАЗАНИЯ. ТАК, В СЕНТЯБРЕ 1715 Г., ПЕТР І ДАЛ ПОРУЧЕНИЕ РОМАНУ ВИЛИМОВИЧУ БРЮСУ « ОСВЕДОМИТЬСЯ, С КОЛИКОГО ЧИСЛА МУЖИКОВ У ШВЕДОВ БЫЛ СОЛДАТ И ПО ЧЕМУ БЫЛО ПОЛОЖЕНО НА ДВОР, ИЛИ НА ГАК, ИЛИ НА ПОГОЛОВЩИНУ, И КАКИМ ОБРАЗОМ ОНЫХ И ИХ ОФИЦЕРОВ ДЕРЖАЛИ НА КВАРТИРАХ?» (ЦИТ. ПО: НИКОЛАЙ ПАВЛОВИЧ ПАВЛОВ-СИЛЬВАНСКИЙ, ПРОЕКТЫ РЕФОРМ В ЗАПИСКАХ СОВРЕМЕННИКОВ ПЕТРА ВЕЛИКОГО: ОПЫТ ИЗУЧЕНИЯ РУССКИХ ПРОЕКТОВ И НЕИЗДАННЫЕ ИХ ТЕКСТЫ, СПБ., 1897, Ч. І, С. 108).

2. «В ПОМЕРАНИИ, В КОЛЬБЕРГЕ И В ДРУГИХ МЕСТАХ СДЕЛАНЫ СОЛДАТЫ ОТ ДЕРЕВЕНЬ ЗА ОБРОКИ СВОЕГО ПАЕЖЮ АЛЬБО УЕЗДУ» (ЦИТ ПО: ПАВЛОВ-СИЛЬВАНСКИЙ, ПРОЕКТЫ РЕФОРМ В ЗАПИСКАХ СОВРЕМЕННИКОВ ПЕТРА ВЕЛИКОГО, С. 10).

3. КАК И ИССЛЕДОВАНИЯ ИСТОРИИ НАЛОГОВОЙ РЕФОРМЫ ПЕТРА ВЕЛИКОГО ВООБЩЕ. В ЭТОМ ОТНОШЕНИИ ФУНДАМЕНТАЛЬНЫЙ ТРУД ЕВГЕНИЯ ВИКТОРОВИЧА АНИСИМОВА ЯВЛЯЕТСЯ ЕДВА ЛИ НЕ ИСКЛЮЧЕНИЕМ. В ОСТАЛЬНЫХ РАБОТАХ, ДАЖЕ В ТАКИХ КЛАССИЧЕСКИХ КАК СОЧИНЕНИЕ ПАВЛА НИКОЛАЕВИЧА МИЛЮКОВА, ВВЕДЕНИЕ ПОДУШНОЙ ПОДАТИ РАССМАТРИВАЕТСЯ В ОБЩЕМ КОНТЕКСТЕ ЭВОЛЮЦИИ ФИНАНСОВ И ФИСКА РОССИИ КОНЦА XVII-XVIII В. СМ. : Е.В. АНИСИМОВ, ПОДАТНАЯ РЕФОРМА ПЕТРА І. ВВЕДЕНИЕ ПОДУШНОЙ ПОДАТИ В РОССИИ 1719-1728 ГГ., Л. : НАУКА, 1982 ; П.Н. МИЛЮКОВ, ГОСУДАРСТВЕННОЕ ХОЗЯЙСТВО РОССИИ В ПЕРВОЙ ЧЕТВЕРТИ ХVІІІ СТОЛЕТИЯ И РЕФОРМА ПЕТРА ВЕЛИКОГО, 1-Е ИЗД., СПБ., 1892. В НАСТОЯЩЕЙ СТАТЬЕ ССЫЛКИ НА МОНОГРАФИЮ П.Н. МИЛЮКОВА ДАЮТСЯ ПО ВТОРОМУ ИЗДАНИЮ 1905 Г.

4. М.М. БОГОСЛОВСКИЙ, ОБЛАСТНАЯ РЕФОРМА ПЕТРА ВЕЛИКОГО. ПРОВИНЦИЯ 1719-1727 ГГ., М., 1902, C. 322-443.

5. ТАМ ЖЕ, С. 404-405.

6. ПСЗ (ПОЛНОЕ СОБРАНИЕ ЗАКОНОВ РОССИЙСКОЙ ИМПЕРИИ), Т. 5, № 3244.

7. БОГОСЛОВСКИЙ, ОБЛАСТНАЯ РЕФОРМА ПЕТРА ВЕЛИКОГО, С. 49.

8. ТАМ ЖЕ.

9. пСЗ, Т. 5, № 3245.

10. ЗДЕСЬ РЕЧЬ ИДЕТ ИМЕННО О ЗЕМСКОМ КОМИССАРЕ - СБОРЩИКЕ ПОДУШНОЙ ПОДАТИ, А НЕ О КАМЕР-КОЛЛЕЖСКОМ ЗЕМСКОМ КОМИССАРЕ - РУКОВОДИТЕЛЕ НИЗШЕЙ АДМИНИСТРАТИВНО-ТЕРРИТОРИАЛЬНОЙ ЕДИНИЦЫ.

11. ПСЗ, Т. 5, № 3294, П. 8, 11, 27, 35.

12. ПСЗ, Т. 5 , № 3295.

13. ПС, Т. 5 , № 3294.

14. ПС, Т. 5 , № 3296.

15. пСз, Т. 5, № 3295, П. 10, 17.

16. пС 3 , Т. 5 , № 3470 .

17. ПСз, Т. 6 , № 3492 .

18. пСз, Т. 6 , № 3687.

19. ПОДРОБНЕЕ О ДЕЯТЕЛЬНОСТИ « ПОНУДИТЕЛЕЙ» ПО ОБЕСПЕЧЕНИЮ ПРОВЕДЕНИЯ ПОДУШНОЙ ПОДАТИ СМ. : Е.В. АНИСИМОВ, ПОДАТНАЯ РЕФОРМА ПЕТРА І, С. 66-68. О ПОНУДИТЕЛЯХ ВООБЩЕ СМ. : Д.О.СЕРОВ, «ПОНУДИТЕЛИ (ИЗ ИСТОРИИ ЧРЕЗВЫЧАЙНОГО КОНТРОЛЯ В РОССИИ ПЕРВОЙ ЧЕТВЕРТИ XVIII В.)», В «МЫ БЫЛИ!» ГЕНЕРАЛ-ФЕЛЬДЦЕЙХМЕЙСТЕР Я.В.БРЮС И ЕГО ЭПОХА: МАТЕРИАЛЫ ВСЕРОССИЙСКОЙ НАУЧНОЙ КОНФЕРЕНЦИИ (12-14 МАЯ 2004 Г.), СПБ., 2004, Ч. ІІ, С. 74-76 ; Д.А. РЕДИН, «РЕВИЗИЯ И. С. АРСЕНЬЕВА: К ВОПРОСУ ОБ ЭВОЛЮЦИИ ЧРЕЗВЫЧАЙНЫХ 
ИНСТИТУТОВ ПЕТРОВСКОГО ВРЕМЕНИ» В ПРОБЛЕМЫ ИСТОРИИ РОССИИ, ВЫП. 7: ИСТОЧНИК И ЕГО ИНТЕРПРЕТАЦИИ, ЕКАТЕРИНБУРГ : ВОЛОТ, С. 139-152.

20. ПСЗ, Т. 6, № 3707.

21. ДОКУМЕНТЫ ПОКАЗЫВАЮТ ГОРАЗДО БОЛЬШЕЕ РАЗНООБРАЗИЕ НАИМЕНОВАНИЙ ЭТИХ ОРГАНОВ : «КАНЦЕЛЯРИЯ ПЕРЕПИСНЫХ ДЕЛ», «КАНЦЕЛЯРИЯ РАСПОЛОЖЕНИЯ ПОЛКОВ», « КАНЦЕЛЯРИЯ РЕВИЗИИ СВИДЕТЕЛЬСТВА МУЖЕСКОГО ПОЛУ ДУШ», « КАНЦЕЛЯРИЯ СЛЕДОВАНИЯ И РЕВИЗИИ», И НАКОНЕЦ, «КАНЦЕЛЯРИЯ ВЕДЕНИЯ ГЕНЕРАЛА-МАЭОРА [БРИГАДИРА, ПОЛКОВНИКА]». ИЗ НОВЕЙШИХ РАБОТ СМ.: М.В. БАБИЧ, ГОСУДАРСТВЕННЫЕ УЧРЕЖДЕНИЯ ХVIII ВЕКА : КОМИССИИ ПЕТРОВСКОГО ВРЕМЕНИ, М. : РОССПЭН, 2003, ОСОБЕННО С. 68-91.

22. РГАДА (РОССИЙСКИЙ ГОСУДАРСТВЕННЫЙ АРХИВ ДРЕВНИХ АКТОВ), Ф.350, ОП. 3, Д.127, Л. 23ОБ. В ЭТОМ ПЕРЕЧНЕ ОТСУТСТВУЕТ АРХАНГЕЛОГОРОДСКАЯ ГУБЕРНИЯ И ПОСЛАННЫЙ ТУДА ПЕРЕПИСЧИК. ИЗ БОЛЕЕ ПОЗДНИХ ДОКУМЕНТОВ ИЗВЕСТНО, ЧТО В РОЛИ ТАКОВОГО В 17241725 ГГ. НА ТЕРРИТОРИИ ГУБЕРНИИ ДЕЙСТВОВАЛ ГЕНЕРАЛ-МАЙОР ЧЕКИН.

23. БОГОСЛОВСКИЙ, ОБЛАСТНАЯ РЕФОРМА ПЕТРА ВЕЛИКОГО, С. 328-389; АНИСИМОВ, ПОДАТНАЯ РЕФОРМА ПЕТРА I, С. 80-100. ЕСЛИ М.М. БОГОСЛОВСКИЙ УДЕЛЯЛ ОСНОВНОЕ ВНИМАНИЕ ВЗАИМООТНОШЕНИЯМ ПЕРЕПИСНЫХ КАНЦЕЛЯРИЙ С МЕСТНЫМИ ВЛАСТЯМИ, ТО Е.В. АНИСИМОВ АКЦЕНТИРОВАЛ ВНИМАНИЕ НА МЕРАХ, ПРЕДПРИНИМАЕМЫХ РЕВИЗОРАМИ-ПЕРЕПИСЧИКАМИ ПО ВЫЯВЛЕНИЮ УТАЙКИ ПЛАТЕЛЬЩИКОВ И НАКАЗАНИЮ ВИНОВНЫХ В ЭТОМ ПОМЕЩИКОВ И ПРЕДСТАВИТЕЛЕЙ ВОТЧИННЫХ АДМИНИСТРАЦИЙ.

24. М.В. БАБИЧ, « ПОЛКОВОЙ ДВОР», ГОСУДАРСТВЕННОСТЬ РОССИИ (КОНЕЦ ХV В. - ФЕВРАЛЬ 1917 Г.) : СЛОВАРЬ-СПРАВОЧНИК. КН. 3 (Л - П), М. : НАУКА, 2001, С. 329.

25. « ПОЛКОВОЕ УПРАВЛЕНИЕ», ВОЕННАЯ ЭНЦИКЛОПЕДИЯ, ПГ., 1915, Т. 18, С. 545-547.

26. псз, т. 7, № 4533, ч. 1, П. 4 ; ч. 2, П. 19.

27. ПСЗ, Т. 7, № 4534, П. 12.1, 12.3, 12.5.

28. ГАТО (ГОСУДАРСТВЕННЫЙ АРХИВ ТЮМЕНСКОЙ ОБЛАСТИ), Ф. И-47, ОП. 1, Д. 4913, Л. 40.

29. ГАТО, Ф. И-47, ОП. 1, Д. 2122, Л. 30.

30. ГАТО, Ф. И-47, ОП. 1, Д. 2122, л. 35.

31. РГАДА, Ф. 1042, ОП. 1, Д. 212, Л. 18.

32. РГАДА, Ф. 987, ОП. 1 , Д. 2, Л. $56,60$.

33. ГАТО, Ф. И-166, ОП. 1 , Д. 5 , Л. 1 ; Д. 6 , л. 27.

34. ГАТО, Ф. И-166, ОП. 1 , Д. 6, Л. 8.

35. ГАТО, Ф. И-181, ОП. 1, Д. 46, Л. 15.

36. ГАТО, Ф. И- 47 , ОП. 1 , Д. 2122 , Л. 22ОБ.

37. ГАТО, Ф. И-166, ОП. 1 , Д. 6, Л. 250.

38. ГАСО (ГОСУДАРСТВЕННЫЙ АРХИВ СВЕРДЛОВСКОЙ ОБЛАСТИ), Ф. 24, ОП. 12, Д. 194, Л. 175 ОБ.

39. ГАСО, Ф. 24, ОП. 12, Д. 194, Л. 1910 Б.

40. ГАТО, Ф. И-166, ОП. 1, Д. 6, Л. 22.

41. НАДО ПРИЗНАТЬСЯ, АВТОР ЭТИХ СТРОК ПЕРВОНАЧАЛЬНО РАЗДЕЛЯЛ ИМЕННО ЭТУ ТОЧКУ ЗРЕНИЯ, АПРИОРИ СЧИТАЯ, ЧТО ПОЛКОВЫЕ ДВОРЫ ЯВЛЯЛИСЬ ВЫШЕСТОЯЩИМИ УЧРЕЖДЕНИЯМИ ПО ОТНОШЕНИЮ К КАНЦЕЛЯРИЯМ ПОДУШНЫХ СБОРОВ - СВОИМ ФИЛИАЛЬНЫМ КОНТОРАМ. СМ. : Д.А. РЕДИН, «ПОЛКОВЫЕ ДИСТРИКТЫ В СИСТЕМЕ МЕСТНОГО ГОСУДАРСТВЕННОГО УПРАВЛЕНИЯ РОССИИ ПЕРВОЙ ТРЕТИ ХVIII ВЕКА (НА ПРИМЕРЕ СИБИРСКОЙ ГУБЕРНИИ)», ПРОБЛЕМЫ СОЦИАЛЬНОЙ И ПОЛИТИЧЕСКОЙ ИСТОРИИ РОССИИ, М. : ИЗД-ВО РАГС, 2009, С. 164, 169.

42. ГАСО, Ф. 24, ОП. 12, Д. 193, Л. 18 ОБ.

43. пСз, т. 7, № 4534, П. 12.

44. ГАТО, Ф. И-166, ОП. 1, Д. 6, Л. 22.

45. БОГОСЛОВСКИЙ, ОБЛАСТНАЯ РЕФОРМА ПЕТРА ВЕЛИКОГО, С. 402.

46. пСз, т. 5, № 3294. П. 8, 11, 27, 35. 
47. В СООТВЕТСТВИИ С П. 4, Ч. 1. « ПЛАКАТА», ПРОВИНИВШИЙСЯ ЗЕМСКИЙ КОМИССАР ПОДЛЕЖАЛ СУДУ ДВОРЯНСКОГО УЕЗДНОГО СЪЕЗДА, ПОДКОНТРОЛЬНОГО КОМАНДОВАНИЮ ПОЛКА (НА ПРАКТИКЕ ЭТИ ПРОЦЕДУРЫ ОКАЗАЛИСЬ ВООБЩЕ В РУКАХ ПЕРЕПИСНЫХ КАНЦЕЛЯРИЙ). НОРМА ГЛАСИЛА, ЧТО В ЭТОМ СЛУЧАЕ НАКАЗАНИЕ ВИНОВНЫХ ОСУЩЕСТВЛЯЛОСЬ «НЕ ОПИСЫВАЯСЬ ГУБЕРНАТОРАМ И ВОЕВОДАМ», ЕСЛИ РЕЧЬ НЕ ШЛА О СОВЕРШЕНИИ ЗЕМСКИМИ КОМИССАРАМИ ТЯЖКИХ ПРЕСТУПЛЕНИЙ, КАРАЕМЫХ СМЕРТЬЮ ИЛИ «ПУБЛИЧНЫМ НАКАЗАНИЕМ» (ЗДЕСЬ ДЕЙСТВОВАЛИ ПОЛНОМОЧИЯ НАДВОРНОГО СУДА, И ЛИШЬ В СЛУЧАЕ ЕГО ОТСУТСТВИЯ ВОЕВОДЫ) : ПСЗ, Т. 7, № 4533. ЯВНО ПРОТИВОРЕЧА « ПЛАКАТУ», « ИНСТРУКЦИЯ ПОЛКОВНИКУ» ОДНОЗНАЧНО ПРЕДПИСЫВАЛА ОТДАВАТЬ ПРОВИНИВШЕГОСЯ КОМИССАРА « ЗЕМСКОМУ СУДУ», « СОГЛАСЯСЬ С ГУБЕРНОТОРОМ ИЛИ ВОЕВОДОЮ» : ПСЗ, Т. 7, № 4534, П. 11.

48. СР., НА ЭТОТ СЧЕТ, ПОЛОЖЕНИЯ « ИНСТРУКЦИИ, ИЛИ НАКАЗА ВОЕВОДАМ» (П. 11) И « ПЛАКАТА» (П. 6). ПСЗ, Т. 5, 3294 ; Т. 7, № 4533.

49. ПСЗ, Т. 5, № 3294, П. 12, 13, 14.

50. пСз, т. 7, № 4534, 4535.

51. псз, т. 7, № 4533, Ч. 2, П. 9, 10, 11, 12, 13, 14, 16, 17.

52. ПСз, Т. 7, № 4535, П. 12, 13.

53. БОГОСЛОВСКИЙ, ОБЛАСТНАЯ РЕФОРМА ПЕТРА ВЕЛИКОГО, С. 394.

54. ПСЗ, Т. 7, № 4533, ч. 2, П. 1 .

55. пСз, т. 7, № 4535, П. 20.

\section{RÉSUMÉS}

L'article examine le statut de l'administration fiscale militaire qui se mit en place en Russie par suite de la réforme fiscale et de l'introduction de la capitation (ou "impôt sur les âmes ») en 1719. Cette réforme eut un grand impact dans les domaines les plus divers de la vie du pays, dont l'organisation de l'administration publique. La collecte de l'impôt capitulaire, destiné à l'entretien de l'armée, fut confiée directement aux régiments, cantonnés dans les provinces. Pour leur permettre de remplir ces fonctions militaro-administratives, Pierre $\mathrm{I}^{\text {er }}$ instaura un système spécial d'organes militaires d'administration dont le déploiement s'effectua dans le contexte d'autres réformes, administratives notamment (réformes collégiale, judiciaire, deuxième réforme du gouvernement local). Dans l'historiographie, il était d'usage de présenter les «cours de régiment" (polkovye dvory) comme des organes de gestion administrative fiscale et militaire. Cependant, sur la base d'une analyse approfondie de textes normatifs et de documents de bureaux, l'auteur démontre le caractère erroné de cette lecture. Après avoir établi que les bureaux de collecte de la capitation constituaient l'instance principale de l'administration militaire et fiscale, il pointe leur place dans le système général des institutions administratives locales et leur position dominante par rapport aux organes de l'administration civile. Tous ces éléments permettent de reconsidérer la conception historiographique courante prônant le caractère «collégial » des réformes administratives des dernières années du règne de Pierre le Grand.

The article examines the status of the fiscal-military administration established following tax reform and the introduction in 1719 of the head, or poll, tax. That reform had a great impact on the most diverse areas of Russian life, including the organization of public administration. The 
poll tax, whose purpose was the maintenance of the army, was collected by regiments garrisoned in the provinces. In order to alleviate the regiments' military-administrative tasks, Peter the Great introduced a special system of military bodies that was deployed within the context of other, mostly administrative (second local government, collegial, and judicial) reforms. Historiography has traditionally held that polkovye dvory were these fiscal-military administrative bodies. However, a thorough analysis of normative texts and tax-bureau records allows the author to demonstrate that historiography has proceeded on erroneous principles. After establishing clearly that the poll-tax collection bureaus were the main bodies handling fiscal-military administration, the author shows their position in the general system of local administrations and their precedence over civil institutions. All these elements allow one to challenge the traditionally held view of the "collegial" character of administrative reforms during Peter the Great's last years.

\section{AUTEUR}

\section{DMITRIJ A. REDIN}

УРАЛЬСКИЙ ФЕДЕРАЛЬНЫЙ УНИВЕРСИТЕТ, ИНСТИТУТ ИСТОРИИ И АРХЕОЛОГИИ УРАЛЬСКОГО

ОТДЕЛЕНИЯ РАН. volot@mail.ru 